\title{
Heme oxygenase-1 orchestrates the immunosuppressive program of tumor-associated macrophages
}

\author{
Emmanuelle Alaluf, ${ }^{1}$ Benoît Vokaer, ${ }^{1}$ Aurélie Detavernier, ${ }^{1}$ Abdulkader Azouz, ${ }^{1}$ \\ Marion Splittgerber, ${ }^{1}$ Alice Carrette, ${ }^{1}$ Louis Boon, ${ }^{2}$ Frédérick Libert, ${ }^{3}$ Miguel Soares, ${ }^{4}$ \\ Alain Le Moine, ${ }^{1}$ and Stanislas Goriely ${ }^{1}$ \\ 'Université Libre de Bruxelles, Institute for Medical Immunology, and ULB Center for Research in Immunology (U-CRI), \\ Gosselies, Belgium. ${ }^{2}$ Bioceros B.V., Utrecht, Netherlands. ${ }^{3}$ Université Libre de Bruxelles, BRICHTcore ULB-VUB and Institute \\ of Interdisciplinary Research in Human and Molecular Biology (IRIBHM), Brussels, Belgium. ${ }^{4}$ Instituto Gulbenkian de \\ Ciência, Oeiras, Portugal.
}

Tumor-associated macrophages (TAMs) contribute to the maintenance of a strong immunosuppressive environment, supporting tumor progression and resistance to treatment. To date, the mechanisms that drive acquisition of these immunosuppressive features are still poorly defined. Heme oxygenase-1 ( $\mathrm{HO}-1)$ is the rate-limiting enzyme that catabolizes free heme. It displays important cytoprotective, antiinflammatory, and antioxidant properties. A growing body of evidence suggests that $\mathrm{HO}-1$ may also promote tumor development. Herein, we show that $\mathrm{HO}-1$ is highly expressed in monocytic cells in the tumor microenvironment (TME) once they differentiate into TAMs. Deletion of HO-1 in the myeloid compartment enhances the beneficial effects of a therapeutic antitumor vaccine by restoring $\mathrm{CD8}{ }^{+} \mathrm{T}$ cell proliferation and cytotoxicity. We further show that induction of $\mathrm{HO}-1$ plays a major role in monocyte education by tumor cells by modulating their transcriptional and epigenetic programs. These results identify $\mathrm{HO}-1$ as a valuable therapeutic target to reprogram the TME and synergize with current cancer therapies to facilitate antitumor response.

Authorship note: BV and AD contributed equally to this work. ALM and SG contributed equally to this work.

Conflict of interest: The authors have declared that no conflict of interest exists.

Copyright: (c) 2020, American Society for Clinical Investigation.

Submitted: September 30, 2019

Accepted: April 30, 2020

Published: May 5, 2020.

Reference information: /CI Insight. 2020;5(11):e133929.

https://doi.org/10.1172/jici.

insight.133929.

\section{Introduction}

Immunotherapy represents a paradigm shift in the treatment of cancer. In the past few years, approaches such as immune checkpoint inhibition or adoptive transfer of engineered $\mathrm{T}$ cells have produced durable responses and long-term survival of many patients for whom previous therapeutic options were ineffective (1-9). These clinical successes demonstrate the essential role of the cancer-immunity interface in tumor progression and therapy. However, only a subset of patients responds to immunotherapies, and some of them acquire resistance to the treatment. The cellular and molecular determinants of responsiveness versus resistance to immunotherapy are incompletely understood $(10,11)$. This may be partly due to the current focus of therapies on the T cell compartment only, with little attention to the side of the antigen-presenting cell. It is likely that a better understanding of how tumors shape their microenvironment, and alter myeloid cell functions, would reveal novel principles of cancer immunotherapy and enable innovative clinical applications, benefiting patients refractory to current immunotherapies $(12,13)$.

In this context, tumor-associated macrophages (TAMs) generally play a deleterious role by supporting multiple aspects of tumor progression (14). They were shown to contribute to the resistance of tumors to biologic therapies, chemotherapies, and radiotherapies through direct trophic support to cancer cells. For example, TAMs may promote angiogenesis, the maintenance of cancer stem cells, and metastatic processes (15). Macrophage infiltration may also interfere with immunotherapy, hampering efforts to reactivate $\mathrm{CD}^{+} \mathrm{T}$ cells by targeting immune checkpoints. Indeed, macrophages express inhibitory receptors (such as programed cell death ligand 1 [PD-L1] or B7-H4) and represent an important source of cytokines (such as IL-10 or TGF- $\beta$ ), chemokines, and enzymes (such as arginase 1 [Arg-1], inducible nitric oxide synthase [iNOS], or indoleamine 2,3-dioxygenase) that contribute to the maintenance of a strong immunosuppressive environment by inhibiting effector $\mathrm{T}$ cells while inducing regulatory $\mathrm{T}$ cells' recruitment and expansion $(16,17)$. 
Heme oxygenase-1 (HO-1) is the rate-limiting enzyme that catalyzes free heme into 3 major biologically active by-products: carbon monoxide, ferrous iron, and biliverdin (converted to bilirubin). In numerous pathological contexts, HO-1 displays important cytoprotective, antiinflammatory, antioxidant, and antiapoptotic properties (18-26). In the context of alloreactivity, we previously demonstrated that HO-1 contributes to the immunosuppressive properties of myeloid cells (27). A growing body of evidence suggests that HO-1 also promotes tumor development. It is expressed in a wide variety of cancers and is generally associated with poor prognosis (28-30). In preclinical models, the administration of HO-1 pharmacologic inhibitors displays antitumor effects $(31,32)$ and improves the response to chemotherapy (33). This effect is at least partially mediated by $\mathrm{CD}^{+} \mathrm{T}$ cells, but the underlying mechanisms of immunomodulation by HO-1 remain unclear (34). Furthermore, expression of HO-1 by TAMs was recently shown to promote transendothelial migration and metastatic spread (35).

Herein, we investigated the role of HO-1 in TAMs. We show that deletion of HO-1 in the myeloid compartment enhances the beneficial effects of a therapeutic antitumor vaccine by restoring $\mathrm{T}$ cell proliferation and cytotoxicity in the tumor microenvironment (TME). We further show that induction of HO-1 plays a major role in monocyte education by tumor cells by modulating their transcriptional and epigenetic programs. Taken together, these results identify HO-1 as a valuable target to reprogram TAMs and improve current strategies of immunotherapy.

\section{Results}

Monocytic cells express HO-1 upon differentiation into macrophages in the TME. In order to evaluate the expression of HO-1 in the myeloid compartment during tumor development, we implanted thymoma cells (EG7OVA) intradermally in C57BL/6 mice because this model was shown to strongly promote the expansion of myeloid suppressive cells (36). We first assessed HO-1 expression in the TME by immunofluorescence staining (Figure 1A and Supplemental Figure 1A; supplemental material available online with this article; https://doi.org/10.1172/jci.insight.133929DS1). We observed HO-1 staining in a portion of CD11 ${ }^{+}$and F4/80+ infiltrating cells. Of note, HO-1 was found in the cytoplasm and/or the nucleus of these cells. This is consistent with previous reports indicating that HO-1 can migrate to the nucleus and modulate transcriptional activity independently of its enzymatic activity (37-39). To further define the cellular sources of HO-1, we evaluated its expression by flow cytometry. Ly6 $\mathrm{C}^{\mathrm{hi}}$ monocytes that are recruited in the TME gradually differentiate into $\mathrm{Ly} 6 \mathrm{C}^{\mathrm{lo}} \mathrm{MHCII}{ }^{+} \mathrm{TAMs}(40)$. The proportion of $\mathrm{Ly}^{6} \mathrm{G}^{\text {hi }}$ granulocytes or LyC6 $^{\text {hi }}$ monocytes $\left(\mathrm{MHCII}^{-}\right.$or $\mathrm{MHCII}^{+}$) expressing HO-1 was low (Figure 1B). In sharp contrast, it was expressed by a significant proportion of $\mathrm{Ly} 6 \mathrm{C}^{\mathrm{lo}} \mathrm{MHCII}{ }^{+} \mathrm{TAMs}$, suggesting that it is part of the program induced in monocytic cells upon exposure to tumor-derived factors. Expression of HO-1 by TAMs was associated with strong expression of classical phenotypic markers of macrophage differentiation, such as F4/80, CD64, CD206, and CD163 (Figure 1C). As compared with HO-1- TAMs, HO-1+ counterparts had decreased MHCII expression. Tumors also influenced myelopoiesis and induced the accumulation

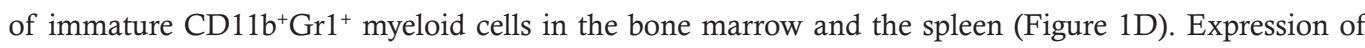
HO-1 in immature CD1 $1 b^{+} \mathrm{Gr}^{+}$myeloid cells from naive and tumor-bearing mice was comparable (Figure $1 \mathrm{E})$. These results indicate that HO-1 expression is specifically induced upon differentiation of monocytic cells in the TME. It was previously shown that STAT3-inducing cytokines, such as IL-6 and IL-10, are potent inducers of HO-1 in macrophages (41). We therefore evaluated the role of these cytokines in our experimental setting using neutralizing antibodies. As shown in Supplemental Figure 1B, HO-1 expression by TAMs was found to be independent of IL-6 and IL-10 signaling, indicating that other tumor-intrinsic factors are responsible for HO-1 induction by the TME. To define the pattern of expression of HO-1 in the context of human cancer, we analyzed single-cell RNA-Seq data from tumor-infiltrating $\mathrm{CD}_{4} 5^{+}$cells collected from patients with breast cancer (42). We observed clear separation of myeloid (characterized by $L Y Z$ and $H L A-D R$ expression) and lymphoid populations (Figure 2). HMOX1 expression was largely restricted to myeloid cells (Figure 2). Next, we identified genes that were upregulated in $H M O X 1^{+}$cells. Many of these genes, such as APOE1, FTH, or FTL1, are characteristic of macrophages or serve as prognostic markers in TAMs (42-44). Gene Ontology analysis revealed several relevant pathways, including the following 3: negative regulation of immune system process, inflammatory response to wounding, and macrophage activation. Trajectory analysis of monocytic clusters identified major components associated with activation and differentiation of macrophages and with monocytes once they enter the TME (42). We identified the cells that exhibited the greatest enrichment for TAMs or monocyte activation signatures and 
A
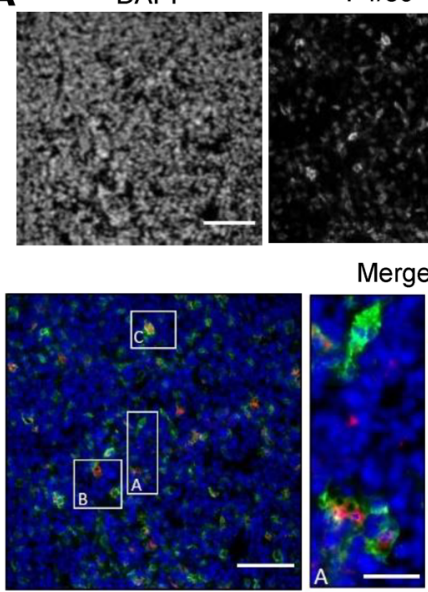

F4/80

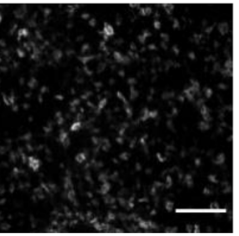

Merge
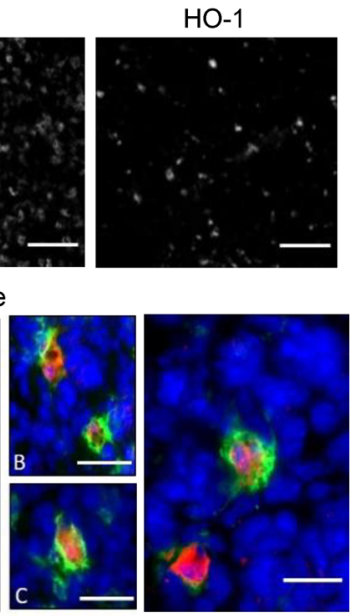

B
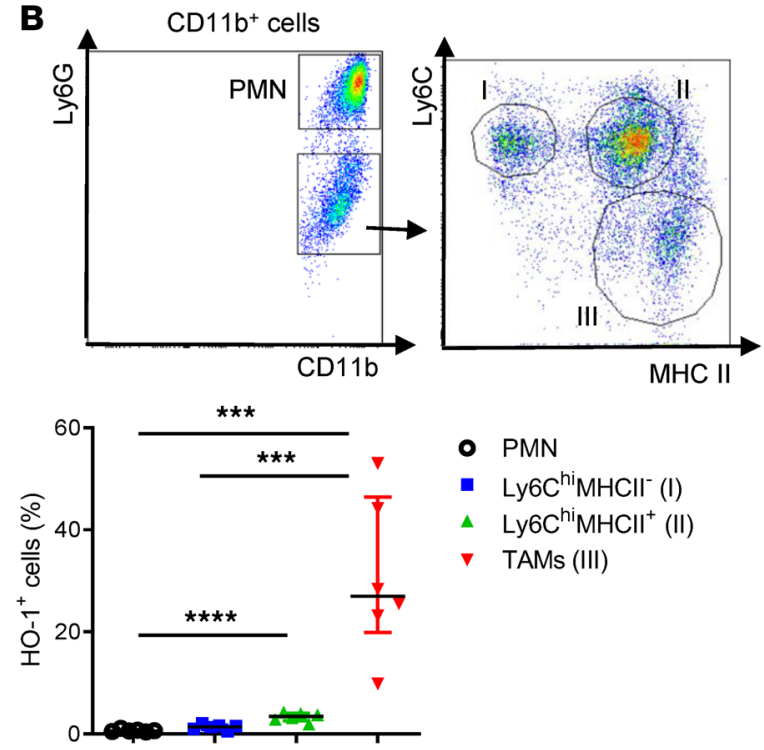

C TAMs
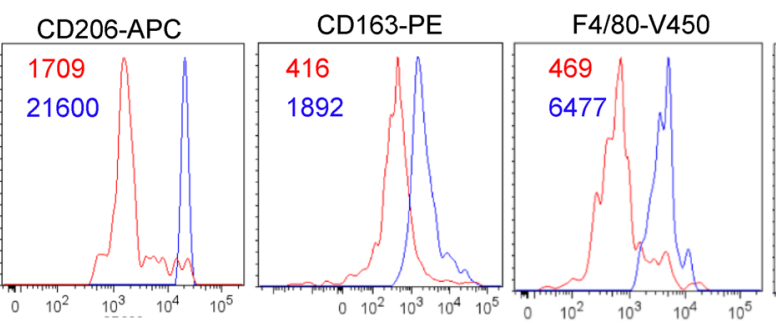

CD64-bv650

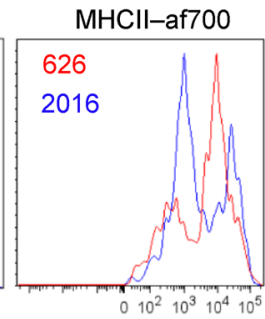

CD11b-bv711
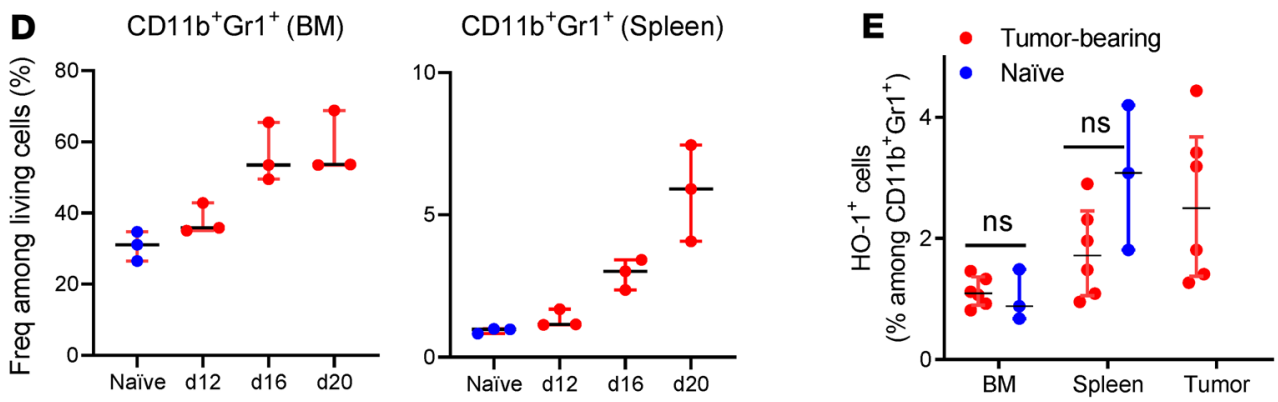

Days after tumor inoculation

Figure 1. HO-1 expression is specifically induced by monocytic cells upon differentiation into macrophages in the TME. (A) HO-1 staining (in red) combined with DAPI costaining showing nuclei (in blue) visualized in tumor slices by immunofluorescence in F4/80+ myeloid cells (in green) in an EG7-OVA tumor 21 days after tumor inoculation in a WT mouse. Scale bar: $5 \mu \mathrm{m}$. (B) Flow cytometry plots pregated on live CD11b $\mathrm{b}^{+}$cells indicate 12 days after tumor inoculation the proportion of

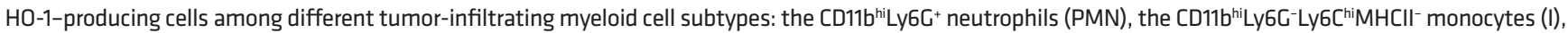

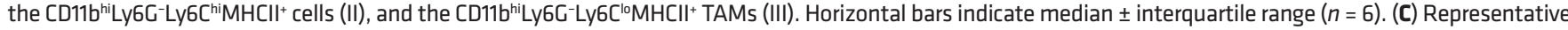
histograms indicating by MFI the level of expression of the specified markers in $\mathrm{HO}^{-1^{+}}$(blue) versus HO-1- (red) TAMs. (D) Representative flow cytometry plots of

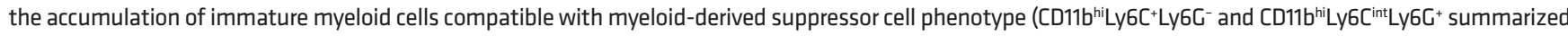
as $\mathrm{CD}_{11} \mathrm{~b}^{+} \mathrm{Gr}^{+}$cells) in the bone marrow (BM) and spleen from tumor-bearing WT mice. Data representative of 3 independent experiments. Each point represents an individual mouse. Horizontal bars indicate median \pm interquartile range (E) $\mathrm{HO}-1$ expression measured by flow cytometry among CD11 $\mathrm{b}^{+} \mathrm{G} \mathrm{r}^{+}$cells from bone marrow, spleen, and EG7-OVA tumor from tumor-bearing WT mice, compared with tumor-free WT mice (naive). Horizontal bars indicate median \pm interquartile; $n=3$ (naive), and $n=6$ (tumor-bearing group). Statistical analysis was performed with Mann-Whitney $U$ test. ${ }^{* *} P<0.001 ;{ }^{* * *} P<0.0001$.

evaluated their expression of HMOX1 (Supplemental Figure 2). These data indicate that HO-1 expression is a feature of monocytes and macrophages once activated in the TME.

Myeloid HO-1 promotes tumor growth by an immunosuppressive mechanism. To determine the impact of HO-1 expression by TAMs on tumor growth, we invalidated Hmox1 in myeloid cells ( $L y s M C r e^{+/ w t}$ $H \operatorname{mox} 1^{1 / / 1} H \operatorname{mox} 1^{1 M}$ mice). We evaluated the growth of intradermally implanted EG7-OVA tumors at 

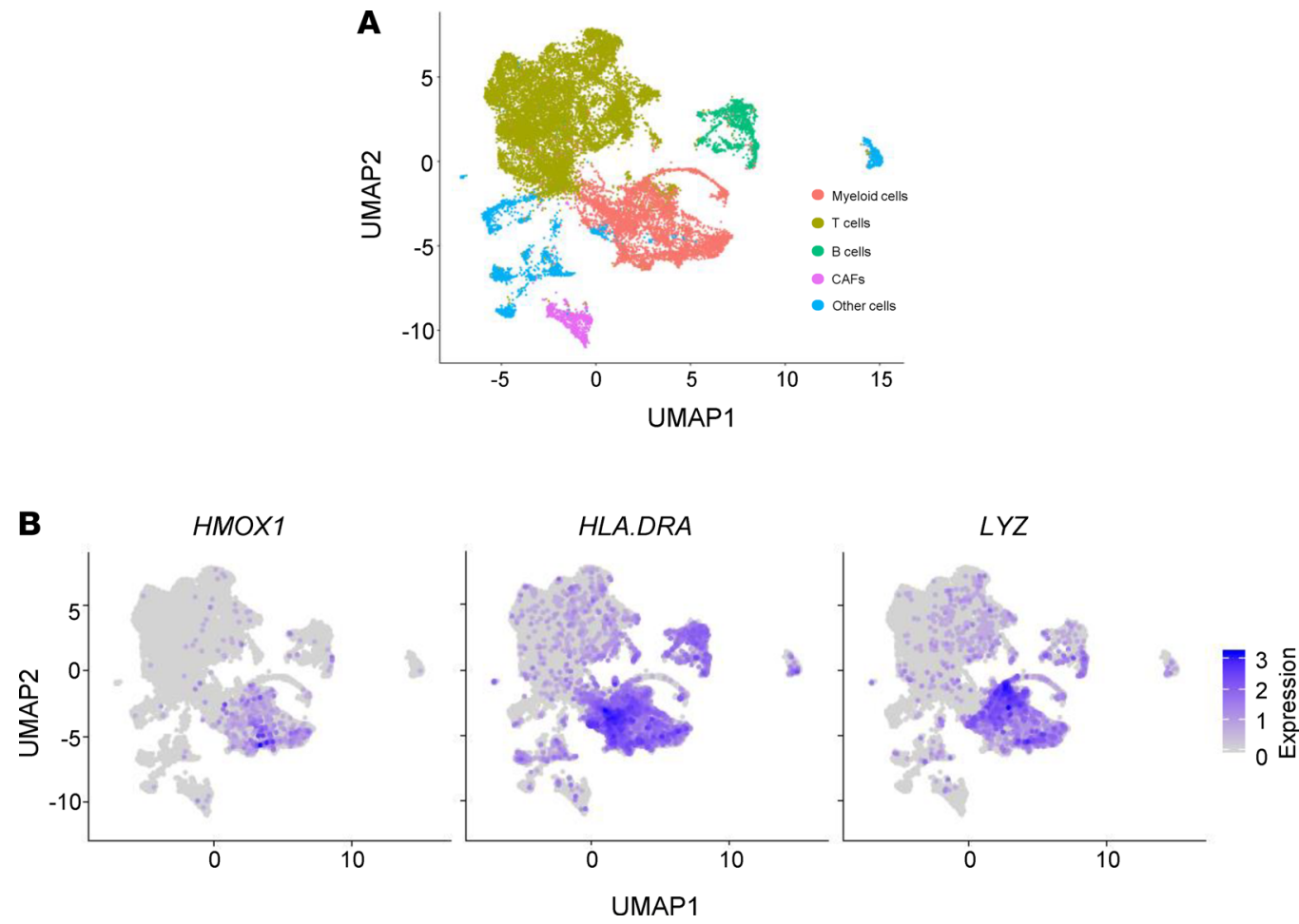

Figure 2. HMOX1 is expressed by myeloid cells that infiltrate human breast tumors. (A) Uniform manifold approximation and projection (UMAP) representation of tumor-infiltrating CD45+ cells from 8 patients with primary breast carcinoma. Each dot represents a single cell colored according to major cell population annotated based on differential gene expression analysis. (B) Normalized expression of HMOX1, HLA-DRA, and LYZ in tumor-infiltrating CD45 cells. Coordinates of cells are the same as in $\mathbf{A}$.

regular intervals. Tumor growth in Hmox $1^{\Delta M}$ mice was comparable to Hmox $1^{\mathrm{A} / \mathrm{fl}}$ littermates (Figure $3 \mathrm{~A}$ ). Next, we repeated these experiments and induced an antitumor $\mathrm{T}$ cell response by immunizing the mice against OVA at day 7 and day 14 postimplantation. As an adjuvant, we used poly(I:C) because this dsR$\mathrm{NA}$ analog was shown to promote $\mathrm{CD} 8^{+} \mathrm{T}$ cell responses in preclinical tumor immunotherapy settings (45). As expected, using this regimen, we observed a delay in tumor growth in $H$ mox $1^{\text {t/fll }}$ mice. However, in most cases, mice had to be sacrificed at later time points because of tumor escape. In contrast, we observed complete tumor regression upon therapeutic immunization in a majority of Hmox $1^{1 M}$ mice. This antitumor effect was abrogated upon depletion of $\mathrm{CD} 8^{+} \mathrm{T}$ cells by antibody treatment (Figure 3B). The effect of HO-1 invalidation in the myeloid compartment on tumor growth was also observed in the absence of immunization, when mice were treated with cyclophosphamide (Supplemental Figure 3), suggesting that HO-1 inhibition could favor antitumor responses in the context of chemotherapy-induced immunogenic cell death. To further evaluate the antigen-specific nature of this enhanced antitumor response, we implanted EG7-OVA tumor cells on one flank and parental EL4 cells on the other flank of the same animal. Upon immunization and transfer of OVA-specific CD8 $8^{+} \mathrm{T}$ cells (OT-1), progression of EG7-OVA tumors was reduced in Hmox $1^{1 M}$ as compared with $H$ mox $1^{\mathrm{B} / \mathrm{fl}}$ mice (Figure 3C). However, in the same animals, growth of EL4 tumors was not restrained in the Hmox $1^{\Delta M}$ group. Taken together, this set of experiments shows that myeloid-specific inactivation of HO-1 potentiates antigen-specific antitumor $\mathrm{CD} 8^{+} \mathrm{T}$ cell responses in the context of therapeutic immunization.

Myeloid HO-1 controls antitumor T cell proliferation and cytotoxicity in the TME. To further assess the effect of myeloid HO-1 on antigen-specific T cell responses, we performed adoptive transfer of CFSE-labeled OT-1 cells 10 days after EG7-OVA implantation. Mice were immunized concomitantly. Two days after, we assessed OT-1 cells' frequency. Although the proportions of OT-1 cells in the spleen or the draining lymph nodes were comparable in both groups, they were strongly increased within the tumors of Hmox $1^{1 M}$ mice as compared with $H$ mox $1^{1 / f l}$ controls (Figure 4A). This was accompanied by high proliferation rate, as assessed by CFSE dilution (Figure 4B) and Ki-67 staining (Figure 4C). Importantly, the proliferation rate of OT-1 cells in the 
A

\begin{tabular}{|c|c|c|c|}
\hline $\begin{array}{c}\text { Tumor } \\
\text { inoculation }\end{array}$ & $\begin{array}{l}\text { OVA+poly(l:C) } \\
\text { immunization }\end{array}$ & $\begin{array}{l}\text { OVA+poly(I:C) } \\
\text { immunization }\end{array}$ & $\rightarrow H \operatorname{mox} 1^{f \mid f l}$ \\
\hline L & $\downarrow$ & $\downarrow$ & $\rightarrow H \operatorname{mox} 1^{\Delta M}$ \\
\hline do & $\mathrm{d} 7$ & & \\
\hline
\end{tabular}
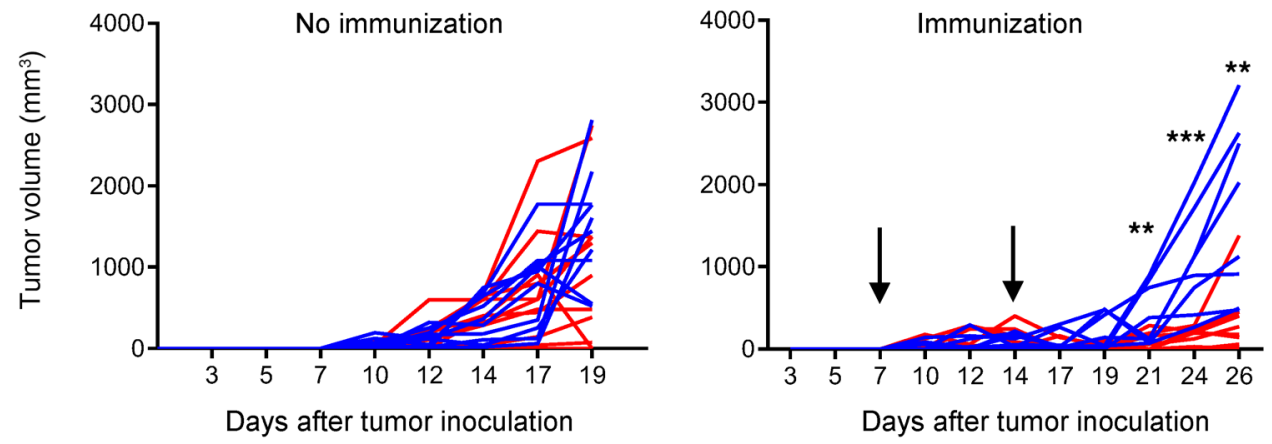

B
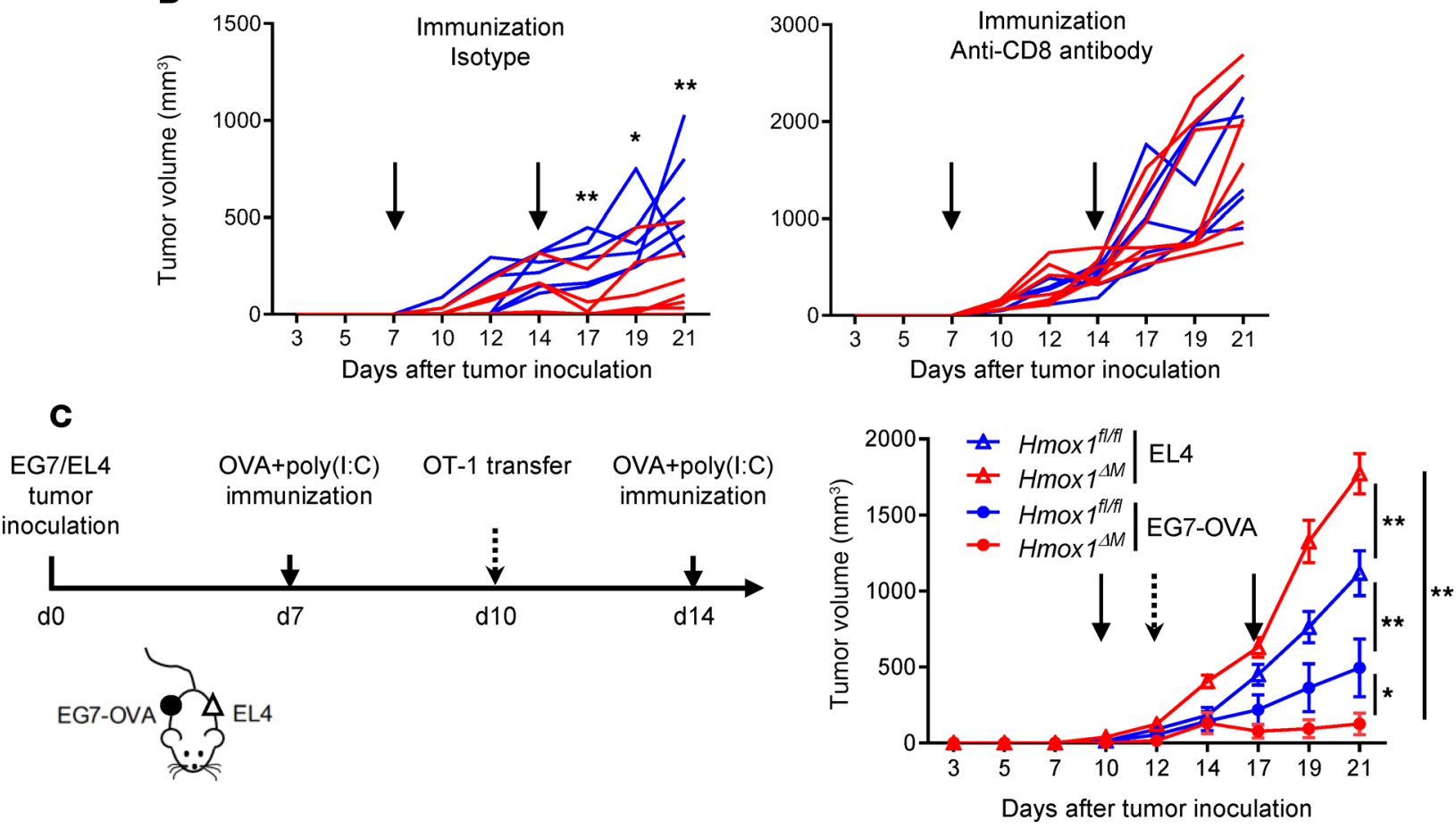

Figure 3. Myeloid H0-1 promotes tumor growth by an immunosuppressive mechanism. EG7-OVA tumor cells were inoculated intradermally at day 0 on the right flank of $H \operatorname{mox}{ }^{1 \mathrm{M}}$ mice $(n=11)$. Their tumor volumes were compared with $H$ mox $7^{f / f 1}$ littermates $(n=10)$ at regular intervals following implantation. (A) There was no significant difference between the groups of tumors. However, a blockade of tumor growth was observed in $\mathrm{Hmox} \mathrm{f}^{\mathrm{MM}}$ mice $(n=11)$ compared with $\mathrm{Hmox} 7^{\mathrm{fl} / \mathrm{fl}}$ littermates $(n=8)$ after therapeutic immunization with subcutaneous injection of ovalbumin protein $(10 \mu \mathrm{g} / \mathrm{mouse})$ and poly(l:C) (50 $\mu \mathrm{g} / \mathrm{mouse}) 7 \mathrm{days}$ after tumor inoculation and boost 7 days later on the right flank of the animals. (B) Intraperitoneal administration of isotype control or CD8 ${ }^{+} T$ cell-depleting monoclonal antibody (clone YTS169) 1 time/wk (500 $\mu$ /mouse). (C) Bilateral tumor model, where EG7-OVA tumor cells were inoculated on the right flank and EL4 cells on the left flank from Hmox $7^{\mathrm{MM}}$ mice $(n=8)$ and $\mathrm{Hmox}^{\mathrm{fl} / \mathrm{fl}}$ mice $(n=10)$ that were therapeutically immunized as described above and adoptively transferred with OTcells $\left(10^{6}\right.$ cells/mouse) at day 10 . Data are representative of 3 independent experiments. Statistical analysis was performed with Mann-Whitney $U$ test ( $\mathbf{A}$ and $\mathbf{B}$ ) and Wilcoxon matched-pairs signed rank test (C). ${ }^{*} P<0.05 ;{ }^{* *} P<0.01 ;{ }^{* *} P<0.001$.

draining lymph nodes and in the spleen was comparable in both experimental groups (Supplemental Figure 4). To evaluate the functionality of adoptively transferred T cells, we analyzed granzyme B and IFN- $\gamma$ expression following ex vivo stimulation of tumor-infiltrating lymphocytes with OVA SIINFEKL peptide. We observed a modest but statistically significant increased expression of these cytotoxic mediators in OT-1 cells transferred in tumor-bearing Hmox $1^{1 M}$ mice (Figure 4, D and E). This was accompanied by higher T-bet levels (Figure 4F). In contrast, expression of Eomes was similar in both groups (Figure 4G). Taken together, these 
A

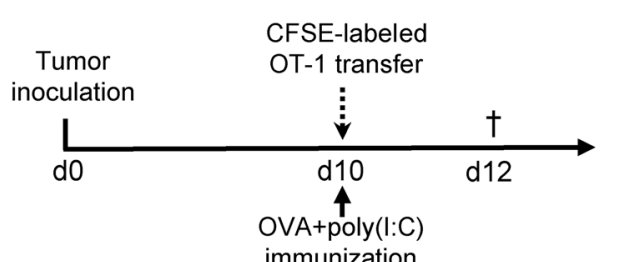

immunization
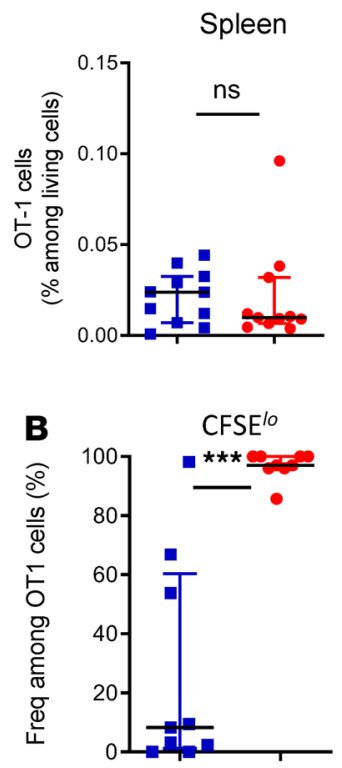

$\mathbf{F}$

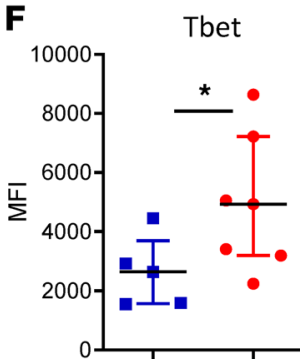

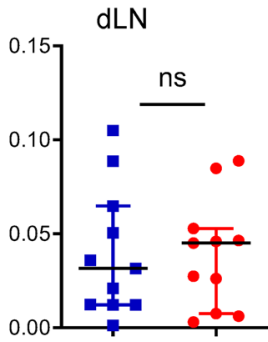

C
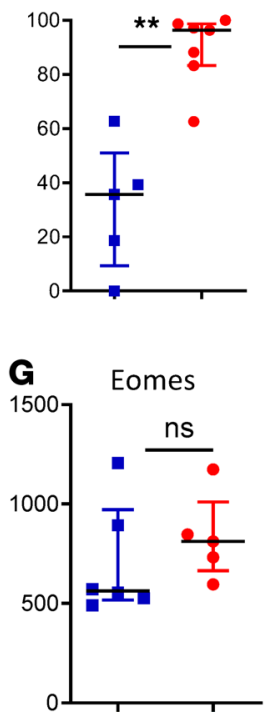

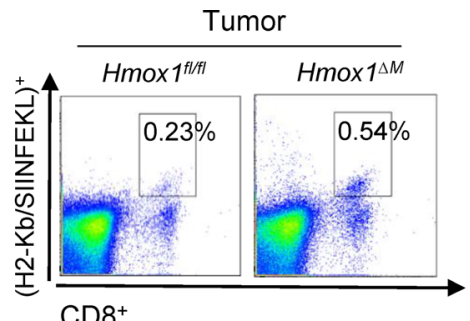

$\mathrm{CD}^{+}$
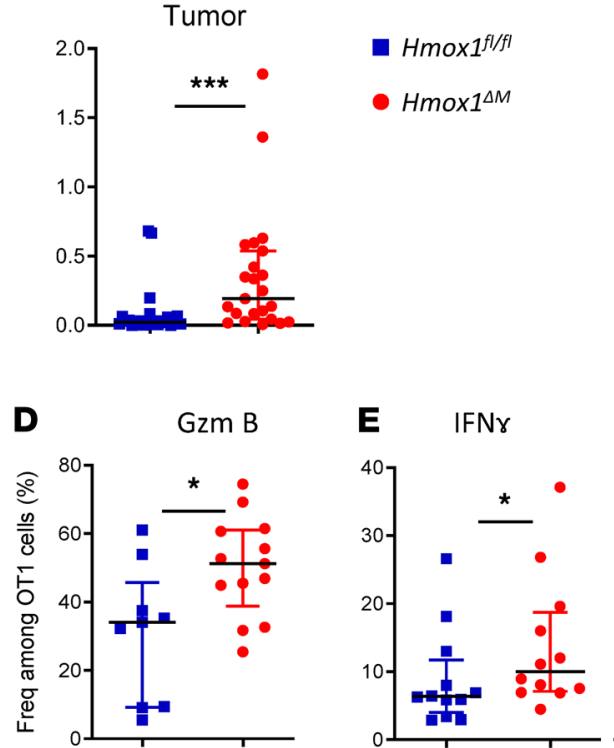

Figure 4. Myeloid HO-1 controls antitumor T cell proliferation and cytotoxicity in the TME. An intravenous adoptive transfer of OT-1 cells $\left(2 \times 10^{6}\right.$ cells/mouse) was performed 10 days after tumor inoculation. This was followed by an immunization with subcutaneous injection of ovalbumin protein (50 $\mu \mathrm{g} /$ mouse) and poly(l:C) (50 $\mu \mathrm{g} / \mathrm{mouse}) 1$ hour later on the right flank of the animals. Two days later, EG7-OVA tumors were enzymatically and mechanistically digested and analyzed by flow cytometry. (A) Proportions of OT-1 cells in the spleen ( $n=11$ and $n=11$ ), the draining lymph nodes $(\mathrm{dLN})(n=11$ and $n=11$ ) (axillary and inguinal lymph nodes on the right side), and the tumor ( $n=24$ and

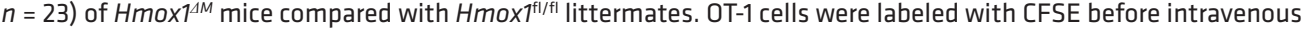
adoptive transfer $\left(2 \times 10^{6}\right.$ cells/mouse). This was followed by an immunization of the mice as described above. (B) Tumor-infiltrating OT-1 cell proliferation assessed by CFSE dilution ( $n=9$ and $n=9$ ) and (C) Ki-67 expression ( $n=5$ and $n$ = 7) among OT-1 cells. (D) Granzyme B (GzmB) ( $n=9$ and $n=13)$ was analyzed by intracytoplasmic staining in tumor-infiltrating OT-1 cells. (E) Production of IFN- $\gamma(n=12$ and $n=12)$ and MFI of (F) T-bet $(n=5$ and $n=7)$ and (G) Eomes ( $n=$ 6 and $n=5$ ) were assessed by ex vivo stimulation overnight with OVA SIINFEKL peptide (and brefeldin A added 2 hours later). Data are pooled from 3/4 experiments. Horizontal bars indicate median \pm interquartile range. Statistical analysis was performed with Mann-Whitney $U$ test. ${ }^{*} P<0.05$; ${ }^{*} P<0.01$; ${ }^{* *} P<0.001$.

data indicate that HO-1 expression by TAMs leads to strong immunosuppressive activity in the TME that limits antigen-specific $\mathrm{CD}^{+} \mathrm{T}$ cell effector function against tumor cells.

HO-1 drives transcriptional and epigenomic programs of TAMs. In order to define the role of HO- 1 in myeloid cells, we examined the proportions of Ly $6 \mathrm{G}^{\text {hi }}$ granulocytes, Ly $6 \mathrm{C}^{\text {hi }}$ monocytes, and Ly $6 \mathrm{C}^{\mathrm{lo}} \mathrm{M}$ $\mathrm{HCII}^{+}$TAMs in tumors of $\operatorname{Hmox}^{4 M}$ and $\operatorname{Hmox}^{\mathrm{fl} / \mathrm{fl}}$ mice 12 days after implantation (Figure 5A). 

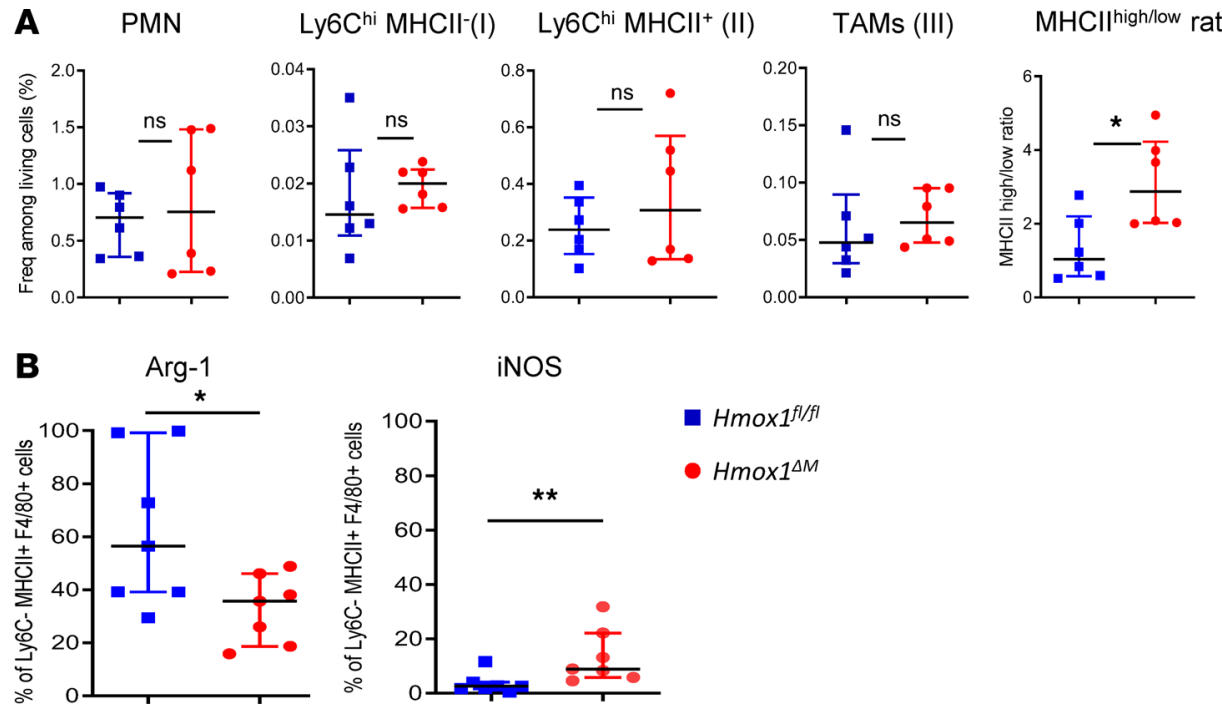

- Hmoxiffffl

- $H \operatorname{mox} 1^{\Delta M}$

Figure 5. H0-1 affects TAM differentiation. EG7-OVA tumor cells were inoculated intradermally at day 0 on the right flank of Hmox $7^{1 \mathrm{M}}$ mice and $H \operatorname{mox} 7^{\mathrm{fl} / \mathrm{fl}}$ littermates. (A) Flow cytometry data showing the frequency of different

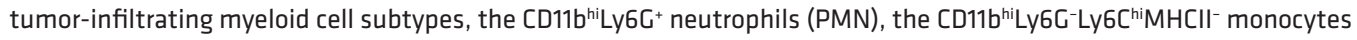

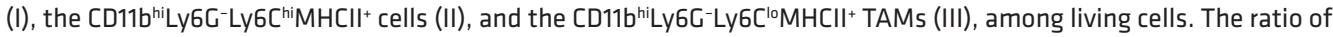

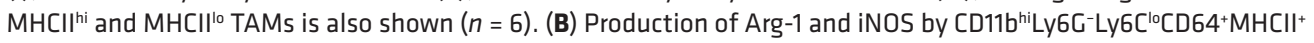
TAMs assessed by flow cytometry intracellular staining, in $\mathrm{Hmox}^{1 \mathrm{M}}$ mice and $\mathrm{Hmox}^{\mathrm{fl} / \mathrm{ff}}$ littermates $(n=7)$, at day 17 [s.c. immunization with OVA $50 \mu \mathrm{g} /$ mouse and poly(I:C) $50 \mu \mathrm{g} /$ mouse at day 12]. Horizontal bars indicate median \pm interquartile range. Statistical analysis was performed with Mann-Whitney $U$ test. ${ }^{*} P<0.05$; ${ }^{* *} P<0.01$.

There were no significant changes in proportions between these 2 groups. However, we observed a larger proportion of higher $\mathrm{MHCII}^{\mathrm{hi}}$ cells in TAMs from Hmox $1^{1 M}$ mice. In addition, myeloid HO-1 deletion led to increased iNOS and decreased Arg-1 expression in TAMs (Figure 5B). Taken together, this suggests that HO-1 could influence their differentiation status, leading to the restoration of tumor-infiltrating $\mathrm{T}$ cell proliferation in $H$ mox $1^{1 M}$ mice. Next, we performed an RNA-Seq experiment on $\mathrm{CD} 11 \mathrm{~b}^{\mathrm{hi}} \mathrm{CD} 64^{+} \mathrm{Ly} 6 \mathrm{C}^{\mathrm{lo}} \mathrm{MHCII}{ }^{+} \mathrm{TAMs}$ from $H \operatorname{mox} 1^{1 M}$ and Hmox $1^{\mathrm{f} / \mathrm{fl}}$ mice. We showed Hmox 1 invalidation through the lack of a full-length RNA of the Hmoxl gene upon LysM-driven expression of Cre recombinase in these cells (Figure 6A) and identified more than 1000 differentially expressed genes (594 down- and 539 upregulated genes in HO-1-deficient cells as compared with their controls; FDR $<0.05$, and fold change $>2$ ) (Figure 6B). We performed gene set enrichment analysis to evaluate the expression of genes that were shown to be upregulated in TAMs as compared with splenic monocytic cells (Figure 6C). We observed a global decrease in the expression of these genes in HO-1-deficient cells, indicating that the core molecular signature the TME induces is dysregulated in the absence of HO-1. The profile observed in HO-1-deficient TAMs did not follow a simple M1/M2 dichotomy because both M1 and M2 signatures were found to be significantly affected. Importantly, multiple genes encoding molecules that participate in the immunosuppressive features of TAMs, such as Arg-1, iNOS, IL-10, PD-L1, and PD-L2, were downregulated in HO-1-deficient cells (Figure 6D). Expression of IL-4-dependent genes was also globally decreased in this group. Furthermore, classical proinflammatory M1 genes, such as $I l 27$ and $I l 12 b$, were also affected (Figure 6D).

To further determine underlying molecular processes at play, we analyzed epigenomic landscapes of these cells by assay for transposase accessible chromatin sequencing (ATAC-Seq) approaches. This technique allows us to map open chromatin regions throughout the genome (46). We observed extensive modifications in HO-1-deficient cells from Hmox $1^{1 M}$ mice. As shown in Figure 7A, 1518 and 4284 regions were found to be significantly more or less accessible in controls, respectively. Most of the differentially accessible peaks were located in enhancers rather than in promoters. We used the Binding and Expression Target Analysis (BETA) package (47) to predict the activating or repressive function of these differentially accessible regions. Regulatory regions that were more/less accessible were clearly associated with genes that were up- or downregulated in WT or HO-1-deficient TAMs, respectively (Figure 7B). This observation indicates that the impact of HO-1 on their transcriptional profile has a 
A

RNA-seq
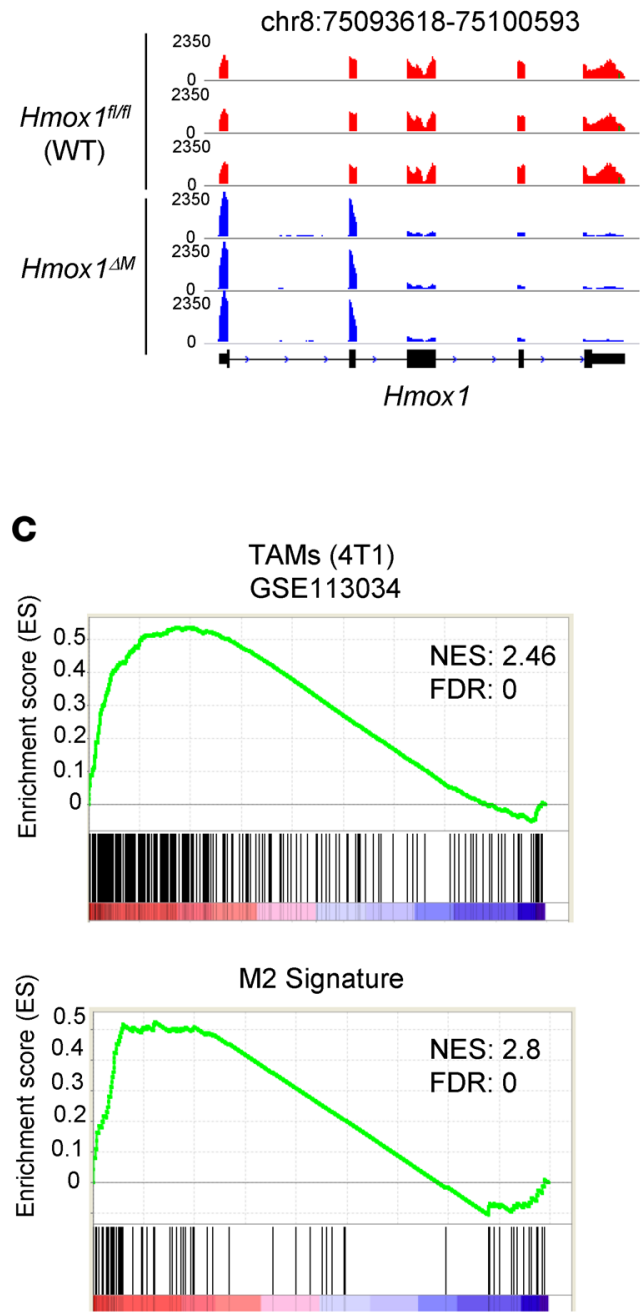

M1 Signature

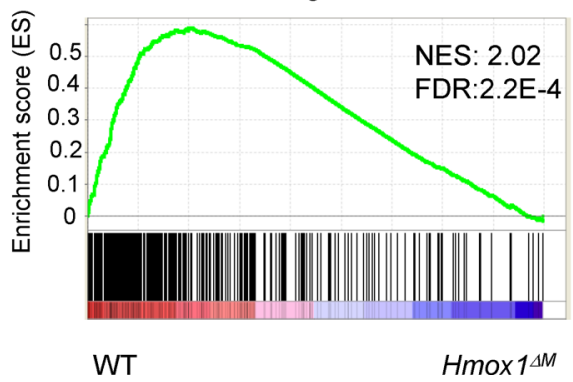

B $\quad$ CD11b ${ }^{\text {hi }}$ Ly6G-Ly6Clow CD64 ${ }^{+} \mathrm{MHCII}^{+}$TAMs

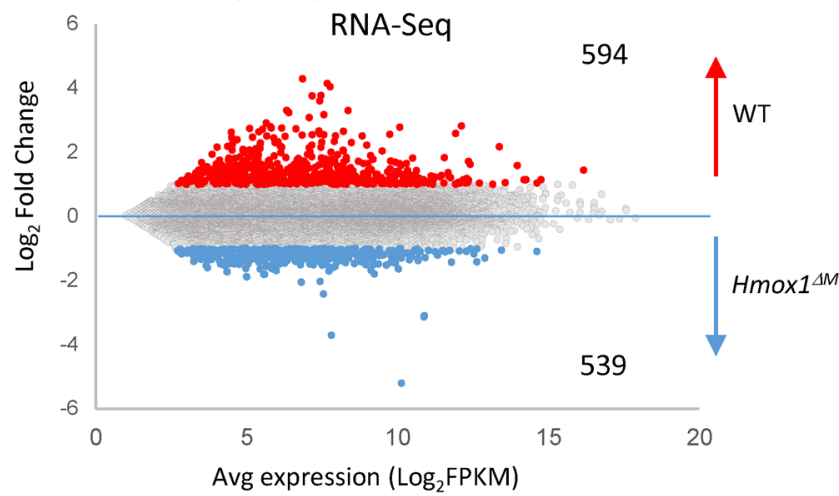

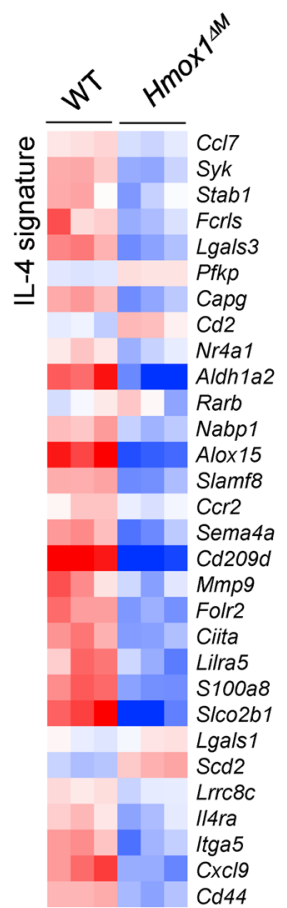

Figure 6. H0-1 drives the transcriptional program of TAMs. (A) Integrative Genomics Viewer tracks showing read coverage for RNA expression of Hmox1 gene in WT (red) and $H_{\operatorname{mox}}{ }^{\mathrm{MM}}$ (blue). Gene position is indicated at the top of the panel. (B) MA plot showing dif-

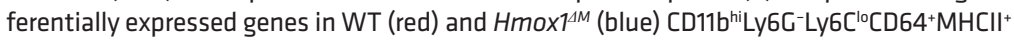
TAMs with the indicated number of genes. (C) Gene set enrichment analysis (GSEA) plots using our RNA-Seq as a data set and the indicated publicly available gene sets. Normalized enrichment score (NES) and FDR are shown. (D) Gene expression heatmap from RNA-Seq data showing the $\log _{2}$ count per million (CPM) of selected pathways.

strong epigenetic component. For example, we observed decreased accessibility in regulatory elements associated with the genes that encode the immune checkpoint molecules PD-L1, PD-L2, and MERTK (Figure 7C) (48), in line with their reduced expression in the absence of HO-1. Similarly, we identified regions that were less accessible within the locus of $\mathrm{Mmp} 2$, which together with other matrix metalloproteinases favors neovascularization and tumor dissemination (49). Next, we performed Gene Ontology analysis using Genomic Regions Enrichment of Annotations Tool (GREAT) (50). The most relevant pathways were associated with regions that were less accessible in HO-1-deficient cells (Figure 8A). As expected, many of these were involved in the regulation of inflammatory response or cytokine signaling. In line with the cytoprotective functions of HO-1 (51), we also observed signatures for wound healing and cell redox homeostasis pathways. Importantly, several metabolic processes were also identified along 
A CD11b hily6G-Ly6Clow $C D 64^{+} \mathrm{MHCll}^{+}$TAMs

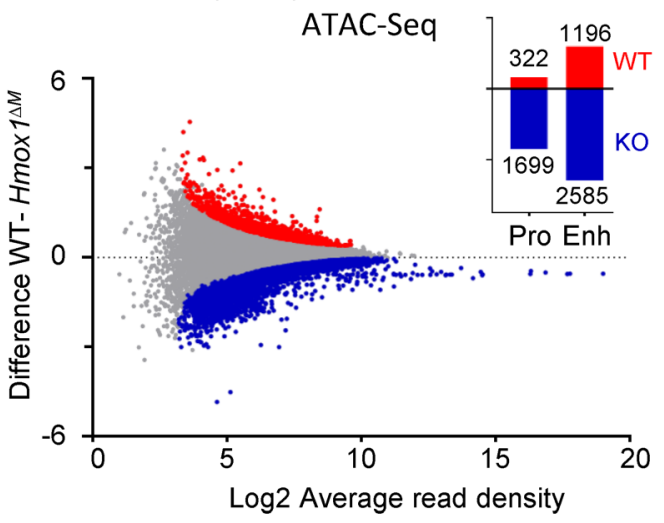

B

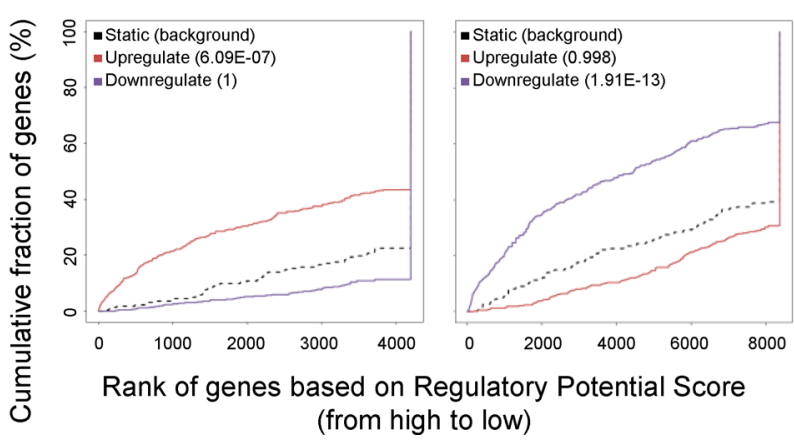

C

chr19:29365746-29474673

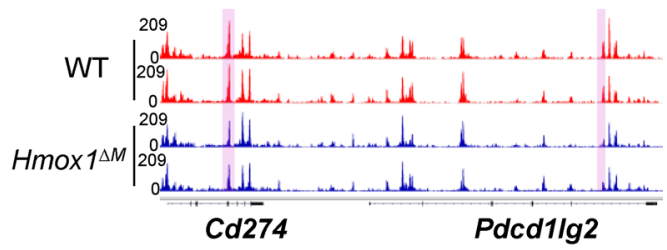

chr2:128695997-128805188

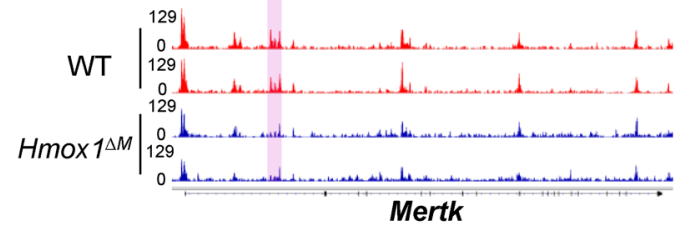

chr8:92826997-92853721

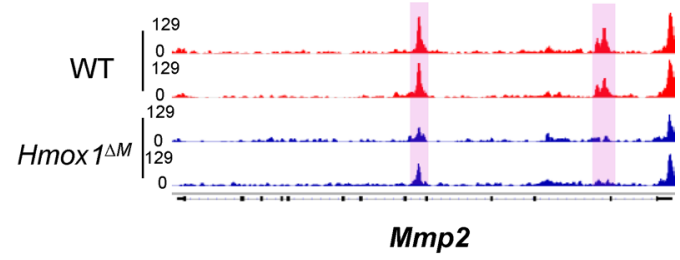

Figure 7. HO-1 drives the epigenomic program of TAMs. (A) MA plot showing $\log _{2}$ average read density of differentially open regions in WT (red) and

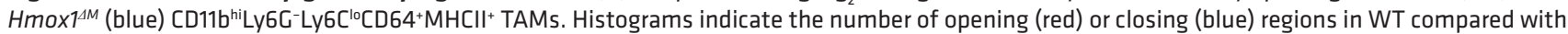
Hmox $7^{1 M}$ cells at promoters (Pro) and enhancers (Enh). (B) Cumulative distribution plot generated by BETA algorithm showing the predicted activating/ repressive functions of differentially open regions in $\mathrm{CD}_{11 b^{\text {hi }}} \mathrm{Ly} 6 \mathrm{C}^{-} \mathrm{Ly} 6 \mathrm{C}^{\mathrm{lo}} \mathrm{CD} 64^{+} \mathrm{MHCII}{ }^{+}$TAMs with the indicated $P$ values determined by the Kolmogorov-Smirnov test. (C) Representative assay for transposase accessible chromatin sequencing (ATAC-Seq) tracks showing enhancers highlighted in purple at the loci of Cd274, Pdcd1lg2, Mertk, and Mmp2. Position of each locus in the genome is indicated at the top of each track.

with other important tumor-related pathways, such as signaling by EGFR, VEGF, and TGF- $\beta$ R (Figure $8 \mathrm{~A})$. These observations suggest important and widespread functional impact of HO-1 on the epigenetic programming of TAMs. We then scanned for binding motifs at the center of ATAC peaks located in these differentially accessible regions using CiiiDER algorithm (52). We observed strong enrichment for consensus binding motifs characteristic of basic region/leucine zipper (bZIP) and zinc finger families of transcription factors in WT and HO-1-deficient cells, respectively (Figure 8B). Among these bZIP factors, we noted motifs for $\mathrm{C} / \mathrm{EBPs}$, which are key factors involved in myeloid cell differentiation, and for Fos- and Jun-related factors. Of note, the consensus antioxidant response elements bound by nuclear factor erythroid 2-related factor 2 (NRF2) were significantly enriched in WT cells. This is of particular interest because it represents the main transcriptional pathway responsible for induction of Hmox1 in response to oxidative stress (53). Conversely, nuclear HO-1 was shown to interact with NRF2 and to promote its transcriptional activity (37). Taken together, these data indicate that HO-1 supports a major transcriptional and epigenetic reprogramming of monocytic cells once they enter the TME.

\section{Discussion}

Tissues' macrophages are highly heterogeneous and plastic and acquire specific functions in response to their environmental cues. In the TME, they integrate multiple signals that reshape their enhancer landscape and as a consequence their transcriptional and functional programs (16). Here we show that HO-1 is induced in monocytic cells that infiltrate the tumor bed upon differentiation into TAMs. Several signals could contribute to this observation. Cytokines such as IL-6 or IL-10, produced by cancer-associated fibroblasts (54), endothelial cells (55), or TAMs themselves (40,56, 57), were shown to be potent inducers of HO-1 as part of a "wound healing" signature (35). However, in our tumor model, HO-1 expression 
A
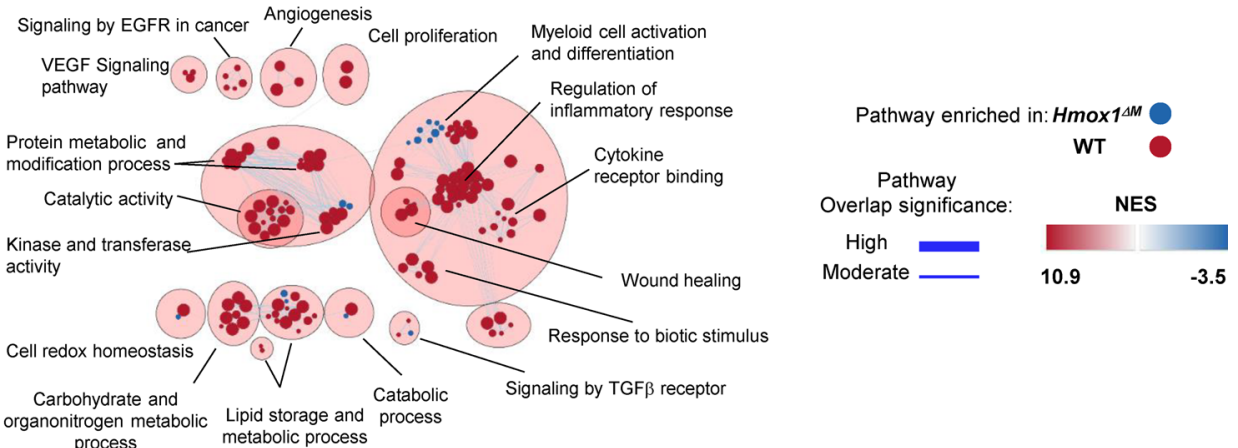

B $\quad H \operatorname{mox} 1^{1 M}$ vs WT

$$
\text { ZF: Sp1,Sp4,Zeb1,KIf }
$$

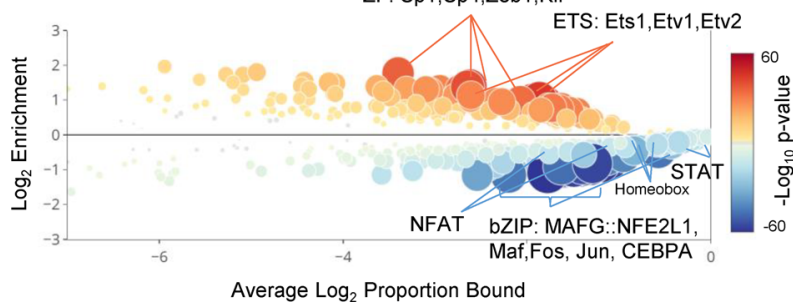

Enriched in WT

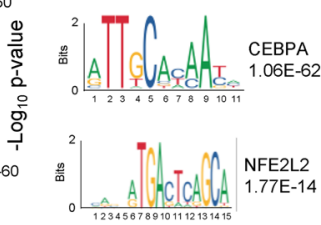

Figure 8. Pathways and motifs associated with differentially accessible regions. (A) Gene set enrichment network displays clusters of redundant pathways overrepresented in WT (red) and Hmox ${ }^{1 \mathrm{M}}$ (blue) TAMs, respectively. Nodes represent gene sets and edges represent mutual overlap. Overlap significance is indicated by the edge's thickness. Color denseness indicates NES. (B) CiiiDER analysis for putative transcription factor motifs from differentially open regions of TAMs in Hmox $1^{1 M}$ and WT. Transcription factors are colored according to their gene coverage $P$ value and whether they are overrepresented (red) or underrepresented (blue). The size of each point is also proportional to $\log _{10} P$ value. Consensus sequence of CCAAT/ enhancer binding protein A (C/EBPA) and NFE2L2 transcription factors are shown with their respective $P$ values.

by TAMs was independent of these signaling pathways. Hence, other signals, such as tissue hypoxia or accumulation of lactic acid, key metabolic features of the TME $(58,59)$, could induce HO-1 through the activation of HIF1 $\alpha$ (60). Intratumor hemorrhage, commonly encountered in cancer, might also lead to extravasation of hemoglobin (61), the physiologic inducer of HO-1.

We demonstrate that myeloid-restricted HO-1 ablation strongly improves the response toward therapeutic immunization by enhancing antitumor $\mathrm{CD}^{+} \mathrm{T}$ cell proliferation and cytotoxicity. In line with this observation, enzymatic inhibition of $\mathrm{HO}-1$ by metalloporphyrins was previously shown to promote tumor regression or complement conventional cancer therapies and to facilitate cytotoxic antitumor immune response $(31-34,62)$. Several lines of evidence in different pathologic conditions suggest that HO-1 induction in macrophages plays a critical role in controlling the adaptive immune response by influencing their polarization (63-69). We observed that HO-1 ablation in TAMs decreased the expression of the M2-associated enzyme arginase 1 while enhancing the expression of iNOS, a classical proinflammatory marker. However, our data indicate that HO-1 ablation had a global impact on the transcriptional and epigenetic profiles of TAMs that is not limited to the classical M1/M2 polarization profile, because we observed decreased expression of both immunosuppressive and proinflammatory markers. This apparent discrepancy could be related to the heterogeneity of TAMs and should be resolved using single-cell-based approaches. Furthermore, it will be important to study these features in different experimental settings that modulate the activation status of TAMs, such as injection of TLR agonists or adoptive cell therapy (70). We suggest that the action of HO-1 on TAMs' differentiation program could be independent of its enzymatic activity because multiple pieces of evidence support a role for HO-1 as a transcriptional modulator. After exposure to hypoxia, HO-1 translocates to the nucleus in a cleaved and enzymatically inactive form, where it directly interacts with transcription factors, such as Nrf2 or JunD, and modulates their activity (37-39). We propose that similar processes could be at play in TAMs and account for their acquisition of an immunosuppressive program. Further understanding of the underlying mechanisms will be important to develop adequate pharmacologic approaches. 
Collectively, our data indicate that HO-1 expression in tumor-infiltrating monocytic cells represents a molecular switch that promotes their immunosuppressive functions. It could therefore represent a valuable target to reprogram the TME and potentially synergize with the current therapeutic approaches focused on the $\mathrm{T}$ cell compartment.

\section{Methods}

Mice. C57BL/6 mice were purchased from Envigo. Hmox $1^{\Delta M}$ mice were generated at the Institute for Medical Immunology by crossing Hmox $1^{\text {fl/fl }}$ mice (in which the Hmox 1 allele was flanked by loxP sites) with LysMCre ${ }^{+/ w t}$ mice, both of which had been backcrossed onto the C57BL/ 6 background for more than 10 generations (Instituto Gulbenkian de Ciência, Oeiras, Portugal). The LysMCre transgene causes a specific deletion of the Hmox1 gene in myeloid cells. Hmox $1^{\mathrm{f} / \mathrm{fl}}$ mice $\left(H m o x 1^{\mathrm{fl} / \mathrm{fl}} L y s M C r e^{w t / w t}\right)$ were used as controls for Hmox $1^{4 M}$ mice and were littermates. MHC class I-restricted, OVA-specific, TCR-transgenic OT-1 mice with a Rag $^{-1-}$ background were obtained from The Jackson Laboratory. All experimental and control mice were 8- to 12-week-old animals and were of the same sex for each experiment.

Tumor cell line. The EL4 lymphoma cell line was obtained from American Type Culture Collection (ATCC, TIB-39). The EG7-OVA tumor cell line was derived from the tumor cell line EL4 (ATCC CRL-2113) by transfection with a plasmid carrying the chicken ovalbumin (OVA) and neomycin phosphotransferase G418 resistance - genes. The cells were maintained at $37^{\circ} \mathrm{C}$ and $5 \% \mathrm{CO}_{2}$ in RPMI 1640 medium (Lonza) supplemented with $10 \%$ fetal calf serum (FCS), $0.1 \mathrm{mM}$ of nonessential amino acids, $100 \mathrm{U} / \mathrm{mL}$ of penicillin, and $100 \mathrm{U} / \mathrm{mL}$ of streptomycin (all reagents from Lonza, hereafter referred to as complete medium). The EG7-OVA tumor cells were cultured in a complete medium supplemented with $1 \mathrm{mg} / \mathrm{mL}$ of G418 sulfate (Geneticin Selective Antibiotic, Thermo Fisher Scientific) once a week. OVA peptide expression on MHCI molecules of EG7-OVA tumor cells was regularly verified by flow cytometry.

Tumor inoculation, therapeutic immunizations, and cytokine neutralization. Tumors were initiated by intradermal injection of $2.5 \times 10^{5}$ tumor cells (in $100 \mu \mathrm{L}$ of sterile PBS) per mouse into the right flank at day 0 . When indicated, at day 7, 10, or 14 after EG7-OVA tumor inoculation, mice were injected subcutaneously with 10 or $50 \mu \mathrm{g}$ of OVA protein (grade VI, A2512, MilliporeSigma) associated with $50 \mu \mathrm{g}$ of poly(I:C) (HMW, VacciGrade, InvivoGen) in $100 \mu \mathrm{L}$ of sterile PBS per mouse on the right flank. Before the immunization, mice were anesthetized intraperitoneally with weight-adjusted amounts of ketamine (1\%, 100 $\mu \mathrm{g} / \mathrm{g}$, Nimatek) and xylazine $(2 \%, 10 \mu \mathrm{g} / \mathrm{g}$, Bayer) solution. In other experiments, the mice were injected i.p. with $0.5 \mathrm{mg}$ of depleting monoclonal antibodies in $200 \mu \mathrm{L}$ of sterile PBS per mouse for CD8 $\alpha^{+} \mathrm{T}$ cell depletion (YTS169, University of Cambridge), 1 day before tumor inoculation and then once a week. The control mice were injected with IgG2b isotype control antibodies (Bio X Cell, clone LTF-2). Peripheral blood samples were collected once a week after depletion and analyzed by flow cytometry to confirm the depletion. For cyclophosphamide experiments, mice were injected i.p. with cyclophosphamide monohydrate (C0768-1G, MilliporeSigma), with a dose of $1.5 \mathrm{mg} /$ mouse in $200 \mu \mathrm{L}$ of PBS at day 10 after tumor inoculation. For cytokine neutralizations, mice were injected every other day, starting at day 1 after tumor inoculation, with $200 \mathrm{ng}$ of anti-IL-6 (clone MP5-20F3, Bio X Cell), anti-IL-10R (clone 1B1.3A, Bio X Cell), or rat IgG1 isotype control (HRPN, Bio X Cell). Mice received 6 doses by i.p. injection.

Tumor monitoring. Mice were monitored every other day for tumor growth by using fine calipers. Tumor volume $\left(\mathrm{mm}^{3}\right)$ is described as $\left(\mathrm{A} \times \mathrm{B}^{2}\right) / 2$, where $\mathrm{A}$ and $\mathrm{B}$ represent tumor length and width, respectively. Mice were sacrificed for tumor analysis when specified or when the total volume of the tumor reached $3000 \mathrm{~mm}^{3}$.

Tissue digestion protocol. Tumors were dissected, finely chopped, and perfused with a digestion solution containing DNAse I $1 \mathrm{mg} / \mathrm{mL}$ (Grade II, MilliporeSigma, 10104159001) $20 \mu \mathrm{L}$ and a mix of collagenase I and II $2.5 \mathrm{mg} / \mathrm{mL}$ (Liberase TL Research Grade, Roche) $20 \mu \mathrm{L}$ in $5 \mathrm{~mL}$ of RPMI 1640 (Lonza) each and were incubated 30 minutes at $37^{\circ} \mathrm{C}$. After $5 \mathrm{~mL}$ of RPMI FCS $5 \%$ with $2 \mathrm{mM}$ ethylenediaminetetraacetic acid (EDTA, MilliporeSigma) were added to each sample, tumor pieces were mashed and filtered, twice. Cell suspensions from spleens were obtained by homogenizing individual spleens to release splenocytes in $5 \mathrm{~mL}$ of RPMI 1640 with $10 \%$ FCS each. The red blood cells were lysed briefly in $800 \mu \mathrm{L}$ of ACK lysis buffer. Lymph node cell suspensions were prepared by dissecting inguinal, deep, and superficial axillary lymph nodes on the right flank and grinding the tissue in RPMI 1640 with 10\% FCS. To obtain bone marrow cell suspensions, femur and tibia from the mice were dissected, rinsed in ethanol, and transferred to RPMI 1640 with $10 \%$ FCS. Bone ends were cut with sterile sharp scissors, and the contents of the bone marrow were flushed with the medium. Bone marrow cells were diluted 
by vigorous pipetting. All the cell suspensions from individual organs were filtered through a $40-\mu \mathrm{m}$ cell strainer, centrifuged at $300 \mathrm{~g}$ for 10 minutes, and resuspended in RPMI 1640 with $10 \%$ FCS.

OT-1 $\mathrm{T}$ cell isolation and adoptive transfer. Cell suspensions from the lymph nodes of the MHC class Irestricted, OVA-specific, TCR-transgenic OT-1 mice (OT-1 cells) ( 8 weeks to 4 months old) were harvested (see tissue digestion protocol) and adoptively transferred by tail vein injection $\left(2 \times 10^{6}\right.$ cells per mouse) on the specified day after tumor inoculation. When specified, OT-1 cells were labeled before intravenous injection with $2 \mu \mathrm{M}$ CFSE (CellTrace CFSE Cell Proliferation Kit, Invitrogen, Thermo Fisher Scientific) by incubating them for 20 minutes at $37^{\circ} \mathrm{C}$ according to the manufacturer's instructions. This was followed by an immunization with OVA protein $(50 \mu \mathrm{g})$ and poly(I:C) $(50 \mu \mathrm{g}) 1$ hour later. Cell division accompanied by CFSE dilution was analyzed by flow cytometry 2 days later (detected in the FITC channel).

Flow cytometry. Cell suspensions were washed, resuspended, and incubated (for 30 minutes at $37^{\circ} \mathrm{C}$ in the dark) in $50 \mu \mathrm{L}$ of PBS with $10 \%$ FCS containing an antibody mix with an Fc-blocking reagent (rat anti-mouse CD16/CD32, BD, clone 2.4G2, dilution 1/200). EDTA, 2 mM, was added to the tumor cell suspensions. The cell surface staining was performed using monoclonal antibodies against the following molecules (clone, company): BV510-conjugated rat anti-mouse CD90.2 (53-2.1, BD), Alexa Fluor 700conjugated rat anti-mouse CD4 (RM 4-5, eBioscience, Thermo Fisher Scientific), Pacific blue-conjugated rat anti-mouse CD8 $\alpha$ (53-6.7, BD), FITC-conjugated hamster anti-mouse TCR $\beta$ (H57-597, BD), PE-conjugated rat anti-mouse CD163 (TNKUPJ, eBioscience, Thermo Fisher Scientific), APC-conjugated rat anti-mouse CD206 (CO68C2, BioLegend), Pacific blue-conjugated rat anti-mouse F4/80 (BM8, eBioscience, Thermo Fisher Scientific), BV650-conjugated mouse anti-mouse CD64 (X54-5/7.1, BD), Alexa Fluor 647-conjugated mouse anti-mouse CD64 (X54-5/7.1, BD), BV711-conjugated rat anti-mouse CD11b (M1/70, BD), Alexa Fluor 700-conjugated rat anti-mouse CD11b (M1/70, BD), BV605-conjugated rat anti-mouse Ly6G (1A8, BioLegend), PE-conjugated rat anti-mouse Ly6G (1A8, BD), PerCP/Cy5.5-conjugated rat anti-mouse Ly6C (HK1.4, BioLegend), Alexa Fluor 700-conjugated MHC class II (I-A/I-E) (M5/114.15.2, eBioscience, Thermo Fisher Scientific), Alexa Fluor 700-conjugated rat anti-mouse IFN- $\gamma$ (XMG1.2, BD), APC-conjugated rat anti-mouse granzyme B (NGZB, eBioscience, Thermo Fisher Scientific), APC-conjugated mouse anti-mouse T-bet (4B10, BioLegend), PE-conjugated anti-mouse Eomes (Dan11mag, eBioscience, Thermo Fisher Scientific), BV605-conjugated rat anti-mouse Ki-67 (16A8, BioLegend), and APC-conjugated mouse anti-mouse OVA 257-264 (SIINFEKL) peptide bound to $\mathrm{H}-2 \mathrm{~Kb}$ (25-D1.16, eBioscience, Thermo Fisher Scientific). Cells were stained to exclude dead cells (LIVE/DEAD Fixable Near-IR Dead Cell Stain Kit, dilution 1/1000, Thermo Fisher Scientific). The intracytoplasmic staining was performed using monoclonal antibodies against PE-Cyanine7-conjugated rat anti-mouse iNOS (CXNFT, eBioscience, Thermo Fisher Scientific ) and APC-conjugated rat anti-human/mouse Arg-1 (A1EXF5, eBioscience, Thermo Fisher Scientific), using the Intracellular Fixation/Permeabilization Buffer Set (eBioscience, Thermo Fisher Scientific, Foxp3/ Transcription Factor Staining Buffer Set, 00-5523-00) according to the manufacturer's instructions. HO-1 intracytoplasmic staining was performed through primary unconjugated mouse anti-mouse HO-1 antibody (ab13248, Abcam) (ab172730, Abcam, for isotype control) and then a secondary FITC-conjugated rat anti-mouse IgG1 (A85-1, BD). When indicated, cells were harvested after density gradient preparation (Lymphoprep) and stimulated ex vivo overnight at $37^{\circ} \mathrm{C}$ with OVA 257-264 SIINFEKL peptide, which consists of 17 15-mer peptides selected for H2-Kb epitope content ( $1 \mu \mathrm{g} / \mathrm{mL}$, Polypeptides Laboratories), in complete medium, in the presence of recombinant human IL-2 $(10 \mathrm{ng} / \mathrm{mL}$, R\&D Systems, Bio-Techne) and brefeldin A (5 $\mathrm{g} / \mathrm{mL}, \mathrm{BD}$ Biosciences) added 2 hours later. IFN- $\gamma$ production was then assessed by $\mathrm{CD}^{+} \mathrm{T}$ cell intracytoplasmic staining. To assess OVA-specific $\mathrm{CD}^{+} \mathrm{T}$ cell response, PE-labeled MHC class I SIINFEKL-specific pentamers (ProImmune) were used. Granzyme B intracytoplasmic staining was used in pentamer ${ }^{+} \mathrm{CD} 8^{+} \mathrm{T}$ cells. Samples were acquired on a BD LSRFortessa flow cytometer. Analyses were performed using FlowJo software (FlowJo LLC).

Immunofluorescence. For imaging, mice were sacrificed 21 days after tumor inoculation. Tumors were dissected, then placed in a solution of OCT embedding medium (Tissue-Tek O.C.T. Compound, Sakura Finetek). OCT-embedded samples were frozen and sectioned on the cryostat microtome (5- $\mu \mathrm{m}$ thickness) and then fixed in methanol. Slides were incubated overnight with a primary antibody mix (FITC-conjugated rat anti-mouse CD11b, clone M1/70, dilution of 1/100, BD; FITC-conjugated rat anti-mouse F4/80, clone BM8, dilution of 1/200, eBioscience, Thermo Fisher Scientific; Texas red-conjugated rabbit anti-mouse HO-1, clone ADISPA-895, dilution of $1 / 100$, Enzo) in the dark at $4^{\circ} \mathrm{C}$, then washed and incubated for 3 hours with a secondary 
antibody mix (FITC-conjugated donkey anti-rat IgG, clone A21208, dilution of 1/200, Invitrogen, Thermo Fisher Scientific; Texas red-conjugated goat anti-rabbit IgG, clone A11006, dilution of 1/150, Invitrogen, Thermo Fisher Scientific). The mix was composed of PBS with $0.1 \%$ of Tween-20 (MilliporeSigma) and 5\% of bovine serum albumin. Nuclear staining was visualized with DAPI (dilution of 1/10,000). Isotype controls $(1 / 100)$ were used instead of primary antibodies to assess the nonspecific background. Images were captured with a ZEISS Axio Observer Z.1 microscope and analyzed with Zen Pro and ImageJ software.

$T A M$ cell sorting. $\mathrm{CD} 11 \mathrm{~b}^{+}$cells were first purified from tumor cell suspensions using positive magnetic selection with a cell isolation kit (Miltenyi Biotec). LIVE/DEAD $\mathrm{CD}^{-} 11 \mathrm{~b}^{+} \mathrm{Ly}_{6} \mathrm{G}^{-} \mathrm{Ly}^{6} \mathrm{C}^{-} \mathrm{MHCII}^{+} \mathrm{CD} 64^{+}$ TAMs were sorted using a BD FACSAria III cell sorter (100,000 cells from Hmox $1^{1 M}$ mice and from WT mice, in triplicates) after surface staining with monoclonal antibody mix (see the section Flow cytometry).

$R N A$-Seq. TAMs were isolated by FACS in RLT buffer and flash-frozen. RNA extraction was performed using RNeasy Mini Kit (QIAGEN), and sample quality was tested on a 2100 Bioanalyzer (Agilent). Libraries were prepared using Ovation SoLo RNA-Seq System (NuGEN Technologies) and underwent paired-end sequencing $\left(25 \times 10^{6}\right.$ paired-end reads/sample, NovaSeq 6000 platform) performed by BRIGHTcore ULBVUB, Belgium (http://www.brightcore.be). Adapters were removed with Trimmomatic-0.36 (with the following parameters: Truseq3-PE.fa:2:30:10 LEADING:3 TRAILING:3 SLIDINGWINDOW:4:15 MINLEN:36 HEADCROP:4) Reads were then mapped to the reference genome mm10 by using STAR_2.5.3 software with default parameters. We then sorted the reads from the alignment according to chromosome positions and indexed the resulting BAM files. Read counts in the alignment BAM files that overlap with the gene features were obtained using HTSeq-0.9.1 with "--nonunique all" option (if the read pair aligns to more than one location in the reference genome, it is counted in all features to which it was assigned and scored multiple times). Genes with no raw read count greater than or equal to 20 in at least 1 sample were filtered out with an $\mathrm{R}$ script, raw read counts were normalized, and a differential expression analysis was performed with DESeq 2 by applying an adjusted $P<0.05$ and an absolute $\log _{2}$ ratio larger than 1 .

ATAC-Seq. ATAC followed by sequencing was performed as following: 20,000 sorted TAMs were collected in $1 \mathrm{~mL}$ of PBS plus $3 \% \mathrm{FBS}$ at $4^{\circ} \mathrm{C}$. Cells were centrifuged, and then cell pellets were resuspended in $50 \mu \mathrm{L}$ of lysis buffer (Tris- $\mathrm{HCl} 10 \mathrm{mM}, \mathrm{NaCl} 10 \mathrm{mM}, \mathrm{MgCl}_{2} 3 \mathrm{mM}$, IGEPAL $0.1 \%$ ) and centrifuged $\left(500 \mathrm{~g}\right.$ ) for 25 minutes at $4^{\circ} \mathrm{C}$. Supernatant was discarded and nuclei were resuspended in $50 \mu \mathrm{L}$ of reaction buffer (Tn5 transposase $2.5 \mu \mathrm{L}$, TD buffer $22.5 \mu \mathrm{L}$, and $\mathrm{H}_{2} \mathrm{O} 25 \mu \mathrm{L}$, Nextera DNA sample preparation kit, Illumina). The reaction was performed for 30 minutes at $37^{\circ} \mathrm{C}$. DNA was purified using the MinElute PCR Purification Kit (QIAGEN). Purified DNA was amplified and indexed by PCR using NEBNext High-Fidelity 2X PCR Master Mix (New England Biolabs) with 10-12 cycles. Amplified libraries were purified using MinElute PCR Purification Kit (QIAGEN), followed by a double AMPURE XP purification (0.5:1 and 1.2:1 ratios), and quality controlled using a Bioanalyzer High-Sensitivity DNA Analysis Kit (Agilent). Paired-end sequencing was performed on NovaSeq platforms (Illumina). Adapters in obtained reads were removed with Trimmomatic 0.36 with the following parameters: Nextera 1.fa:1:25:6 LEADING:3 TRAILING:3 SLIDINGWINDOW:4:15 MINLEN:36. Paired-end reads were mapped to mouse genome mm10 with Bowtie2 $(71,72)$ using the following parameters for paired-end reads: $-\mathrm{X} 2000$-fr -very-sensitive -no-discordant -no-mixed -non-deterministic. Reads from the alignment were sorted and indexed according to chromosomes. Reads located within the blacklist of the ENCODE project 46 were then removed. Duplicate reads were removed with MarkDuplicates tools (Picard suite). Peaks were called with MACS2 (73) using the following parameters: -f BAMPE -g mm -q 0.05 --nomodel --call-summits -B -SPMR.

Regions obtained by MACS2 were subjected to differential analysis using DESeq2 provided by SeqMonk 1.43.0 (Mapped Sequence Analysis Tool, Babraham Bioinformatics, http://www.bioinformatics.babraham. ac.uk/projects/seqmonk/). First, we created an atlas containing all obtained peaks for all the populations using bedtools (74) with a minimum overlapping of $1 \mathrm{bp}$. We used DESeq2 (75) with a $P$-adjusted cutoff of 0.05 . Resulting peaks were separated into 2 categories: peaks located in promoters (located within $2 \mathrm{~kb}$ around the transcription start site) and peaks located in enhancers (not located in the defined promoter regions). For downstream visualization, a scaling factor was calculated using deepTools package (76) to normalize peak intensity to fraction of reads in peaks and generate bigWig files. For Gene Ontology analysis, we introduced BED files from differential ATAC-Seq peaks to GREAT with default parameters (50). For motif analysis, CiiiDER algorithm was used to perform motif enrichment in the differentially accessible regions. We used BETA package with default parameters to integrate ATAC-Seq (differentially accessible regions) and RNA-Seq (transcriptome) data and evaluate the regulatory potential of chromatin accessibility to promote/repress genes' expression. 
Single-cell RNA-Seq analysis. Preprocessed transcription-counts matrices from tumor-infiltrating CD45 cells of 8 primary breast carcinomas were downloaded in duplicates, resulting in 21,346 cells, and loaded into R to be analyzed using Seurat package (version 3.1.2) (77). SCTransform function with default parameters was used for normalizing, scaling, and finding variable features among cells. Principal component analysis was performed with default parameters and used in UMAP analysis to identify clusters. Cell populations were identified by performing differential expression analysis between clusters (related to Figure 2). To identify cells expressing HO-1, we annotated cells with HMOX1>0 counts as positive (535 cells) and $H M O X 1=0$ count as negative (20,811 cells). We performed a new differential expression analysis between $\mathrm{HMOX}^{+}$and $\mathrm{HMOX1}^{-}$clusters to identify genes associated with $\mathrm{HO}-1$ expression (related to Supplemental Figure 2). We used AUCell R package (78) to score the activity of gene sets in each cell. We surveyed 21,346 cells to score gene sets that include genes highly correlated with TAMs' activation and monocytes' activation (42). Threshold of the area under the recovery curve was set to 0.2 and 0.3 for the TAM activation component and the monocyte activation component, respectively.

Data availability. RNA-Seq and ATAC-Seq data that support the findings reported in this study have been deposited in the National Center for Biotechnology Information's Gene Expression Omnibus database with the accession code GSE148585.

Statistics. All data points were included with median and interquartile range. A 2-tailed nonparametric Mann-Whitney $U$ test was used to compare 2 data sets and a Wilcoxon matched-pairs signed-rank test when different tumors from the same animal were compared. Differences were considered statistically significant as follows: $P$ values less than 0.05 were flagged with ${ }^{*}$, less than 0.01 with **, less than 0.001 with $* * *$, and less than 0.0001 with ${ }^{* * * *}$. NS means not statistically significant. All graphs were created and statistical analyses were performed using GraphPad Prism 6 software.

Study approval. All animal studies were approved by the Animal Welfare and Ethics Committee of the Université Libre de Bruxelles Institute of Molecular Biology and Medicine. All experiments were conducted in accordance with recommended guidelines and regulations.

\section{Author contributions}

EA conducted most of the experiments. BV, AD, AA, and AC contributed to some experiments. AA, M Splittgerber, and FL performed bioinformatics analysis. EA, AD, AA, and BV analyzed the data and prepared the figures. M Soares and LB provided critical reagents. ALM and SG supervised the work and wrote the manuscript. All authors were involved in critically revising the manuscript for important intellectual content. All authors had full access to the data and approved the manuscript before it was submitted by the corresponding author.

\section{Acknowledgments}

The authors wish to thank Muriel Moser for helpful discussions. This study was supported by the Fonds National de la Recherche Scientifique (FRS-FNRS, Belgium), the European Regional Development Fund of the Walloon Region (Wallonia-Biomed portfolio, 411132-957270), the IMMUCAN project (Walloon Region), the Fonds Erasme (Erasme Hospital, Université Libre de Bruxelles), the Fondation Rose et Jean Hoguet, the Fonds Lekime-Ropsy, the Fonds David et Alice Van Buuren, and the Fondation Jaumotte-Demoulin. SG is a senior research associate of the FRS-FNRS.

Address correspondence to: Stanislas Goriely, Institute for Medical Immunology, 8 rue Adrienne Bolland, B-6041, Charleroi-Gosselies, Belgium. Phone: 32.2.650.9588; Email: stgoriel@ulb.ac.be.

\footnotetext{
1. Schadendorf D, et al. Pooled analysis of long-term survival data from phase II and phase III trials of ipilimumab in unresectable or metastatic melanoma. J Clin Oncol. 2015;33(17):1889-1894.

2. Larkin J, et al. Combined nivolumab and ipilimumab or monotherapy in untreated melanoma. N Engl J Med. 2015;373(1):23-34.

3. Motzer RJ, et al. Nivolumab plus ipilimumab versus sunitinib in advanced renal-cell carcinoma. $N$ Engl J Med. 2018;378(14):1277-1290.

4. Overman MJ, et al. Nivolumab in patients with metastatic DNA mismatch repair-deficient or microsatellite instability-high colorectal cancer (CheckMate 142): an open-label, multicentre, phase 2 study. Lancet Oncol. 2017;18(9):1182-1191.

5. Antonia SJ, et al. Nivolumab alone and nivolumab plus ipilimumab in recurrent small-cell lung cancer (CheckMate 032): a multicentre, open-label, phase 1/2 trial. Lancet Oncol. 2016;17(7):883-895.

6. Hellmann MD, et al. Nivolumab plus ipilimumab in lung cancer with a high tumor mutational burden. $N$ Engl J Med.
} 
2018;378(22):2093-2104.

7. Brentjens RJ, et al. CD19-targeted T cells rapidly induce molecular remissions in adults with chemotherapy-refractory acute lymphoblastic leukemia. Sci Transl Med. 2013;5(177):177ra38.

8. Schuster SJ, et al. Tisagenlecleucel in adult relapsed or refractory diffuse large B-cell lymphoma. NEngl J Med. 2019;380(1):45-56.

9. Rapoport AP, et al. NY-ESO-1-specific TCR-engineered T cells mediate sustained antigen-specific antitumor effects in myeloma. Nat Med. 2015;21(8):914-921.

10. Messmer MN, Netherby CS, Banik D, Abrams SI. Tumor-induced myeloid dysfunction and its implications for cancer immunotherapy. Cancer Immunol Immunother. 2015;64(1):1-13.

11. Munn DH, Bronte V. Immune suppressive mechanisms in the tumor microenvironment. Curr Opin Immunol. 2016;39:1-6.

12. Friedrich M, et al. Tumor-induced escape mechanisms and their association with resistance to checkpoint inhibitor therapy. Cancer Immunol Immunother. 2019;68(10):1689-1700.

13. Kalathil SG, Thanavala Y. High immunosuppressive burden in cancer patients: a major hurdle for cancer immunotherapy. Cancer Immunol Immunother. 2016;65(7):813-819.

14. Yang M, McKay D, Pollard JW, Lewis CE. Diverse functions of macrophages in different tumor microenvironments. Cancer Res. 2018;78(19):5492-5503.

15. Granot Z, Fridlender ZG. Plasticity beyond cancer cells and the "immunosuppressive switch". Cancer Res. 2015;75(21):4441-4445.

16. DeNardo DG, Ruffell B. Macrophages as regulators of tumour immunity and immunotherapy. Nat Rev Immunol. 2019;19(6):369-382.

17. Noy R, Pollard JW. Tumor-associated macrophages: from mechanisms to therapy. Immunity. 2014;41(1):49-61.

18. Bolisetty S, Zarjou A, Agarwal A. Heme oxygenase 1 as a therapeutic target in acute kidney injury. Am J Kidney Dis. 2017;69(4):531-545.

19. Chang M, Xue J, Sharma V, Habtezion A. Protective role of hemeoxygenase-1 in gastrointestinal diseases. Cell Mol Life Sci. 2015;72(6):1161-1173.

20. Cheng Y, Rong J. Therapeutic potential of heme oxygenase-1/carbon monoxide system against ischemia-reperfusion injury. Curr Pharm Des. 2017;23(26):3884-3898.

21. Zhang MM, et al. Heme oxygenase-1 gene promoter polymorphisms are associated with coronary heart disease and restenosis after percutaneous coronary intervention: a meta-analysis. Oncotarget. 2016;7(50):83437-83450.

22. Buechler C, Pohl R, Aslanidis C. Pro-resolving molecules-new approaches to treat sepsis? Int J Mol Sci. 2017;18(3):E476.

23. Schumacher A, Zenclussen AC. Effects of heme oxygenase-1 on innate and adaptive immune responses promoting pregnancy success and allograft tolerance. Front Pharmacol. 2014;5:288.

24. Li BZ, et al. Therapeutic potential of HO-1 in autoimmune diseases. Inflammation. 2014;37(5):1779-1788.

25. Zhou H, Ying X, Liu Y, Ye S, Yan J, Li Y. Genetic polymorphism of heme oxygenase 1 promoter in the occurrence and severity of chronic obstructive pulmonary disease: a meta-analysis. J Cell Mol Med. 2017;21(5):894-903.

26. Lever JM, Boddu R, George JF, Agarwal A. Heme oxygenase-1 in kidney health and disease. Antioxid Redox Signal. 2016;25(3):165-183.

27. De Wilde V, et al. Endotoxin-induced myeloid-derived suppressor cells inhibit alloimmune responses via heme oxygenase-1. Am J Transplant. 2009;9(9):2034-2047.

28. Nitti M, Piras S, Marinari UM, Moretta L, Pronzato MA, Furfaro AL. HO-1 induction in cancer progression: a matter of cell adaptation. Antioxidants (Basel). 2017;6(2):E29.

29. Chau LY. Heme oxygenase-1: emerging target of cancer therapy. J Biomed Sci. 2015;22:22.

30. Cerny-Reiterer S, et al. Identification of heat shock protein 32 (Hsp32) as a novel target in acute lymphoblastic leukemia. Oncotarget. 2014;5(5):1198-1211.

31. Cheng CC, et al. Blocking heme oxygenase-1 by zinc protoporphyrin reduces tumor hypoxia-mediated VEGF release and inhibits tumor angiogenesis as a potential therapeutic agent against colorectal cancer. J Biomed Sci. 2016;23:18.

32. Fang J, et al. In vivo antitumor activity of pegylated zinc protoporphyrin: targeted inhibition of heme oxygenase in solid tumor. Cancer Res. 2003;63(13):3567-3574.

33. Abdalla MY, et al. Enhancing responsiveness of pancreatic cancer cells to gemcitabine treatment under hypoxia by heme oxygenase-1 inhibition. Transl Res. 2019;207:56-69.

34. Arnold JN, Magiera L, Kraman M, Fearon DT. Tumoral immune suppression by macrophages expressing fibroblast activation protein- $\alpha$ and heme oxygenase-1. Cancer Immunol Res. 2014;2(2):121-126.

35. Muliaditan T, et al. Macrophages are exploited from an innate wound healing response to facilitate cancer metastasis. Nat Commun. 2018;9(1):2951.

36. Youn JI, Nagaraj S, Collazo M, Gabrilovich DI. Subsets of myeloid-derived suppressor cells in tumor-bearing mice. J Immunol. 2008;181(8):5791-5802.

37. Biswas C, et al. Nuclear heme oxygenase-1 (HO-1) modulates subcellular distribution and activation of Nrf2, impacting metabolic and anti-oxidant defenses. J Biol Chem. 2014;289(39):26882-26894.

38. Hsu FF, Chiang MT, Li FA, Yeh CT, Lee WH, Chau LY. Acetylation is essential for nuclear heme oxygenase-1-enhanced tumor growth and invasiveness. Oncogene. 2017;36(49):6805-6814.

39. Lin Q, et al. Heme oxygenase-1 protein localizes to the nucleus and activates transcription factors important in oxidative stress. J Biol Chem. 2007;282(28):20621-20633.

40. Movahedi K, et al. Different tumor microenvironments contain functionally distinct subsets of macrophages derived from Ly6C(high) monocytes. Cancer Res. 2010;70(14):5728-5739.

41. Ricchetti GA, Williams LM, Foxwell BM. Heme oxygenase 1 expression induced by IL-10 requires STAT-3 and phosphoinositol-3 kinase and is inhibited by lipopolysaccharide. J Leukoc Biol. 2004;76(3):719-726.

42. Azizi E, et al. Single-cell map of diverse immune phenotypes in the breast tumor microenvironment. Cell. 2018;174(5):1293-1308.e36.

43. Tang X. Tumor-associated macrophages as potential diagnostic and prognostic biomarkers in breast cancer. Cancer Lett. 2013;332(1):3-10.

44. Wang W, et al. Ferritin H is a novel marker of early erythroid precursors and macrophages. Histopathology. 2013;62(6):931-940. 
45. Perret R, Sierro SR, Botelho NK, Corgnac S, Donda A, Romero P. Adjuvants that improve the ratio of antigen-specific effector to regulatory T cells enhance tumor immunity. Cancer Res. 2013;73(22):6597-6608

46. Buenrostro JD, Wu B, Chang HY, Greenleaf WJ. ATAC-seq: a method for assaying chromatin accessibility genome-wide. Curr Protoc Mol Biol. 2015;109:21.29.1-21.29.9.

47. Wang S, et al. Target analysis by integration of transcriptome and ChIP-seq data with BETA. Nat Protoc. 2013;8(12):2502-2515.

48. Akalu YT, Rothlin CV, Ghosh S. TAM receptor tyrosine kinases as emerging targets of innate immune checkpoint blockade for cancer therapy. Immunol Rev. 2017;276(1):165-177.

49. Deryugina EI, Quigley JP. Tumor angiogenesis: MMP-mediated induction of intravasation- and metastasis-sustaining neovasculature. Matrix Biol. 2015;44-46:94-112.

50. McLean CY, et al. GREAT improves functional interpretation of cis-regulatory regions. Nat Biotechnol. 2010;28(5):495-501.

51. Poss KD, Tonegawa S. Reduced stress defense in heme oxygenase 1-deficient cells. Proc Natl Acad Sci U S A. 1997;94(20):10925-10930.

52. National Institutes of Health. rs161275 Reference Report. National Institutes of Health website. https://www.ncbi.nlm.nih. gov/snp/rs161275. Published July 9, 2019. Accessed May 5, 2020.

53. Alam J, Cook JL. Transcriptional regulation of the heme oxygenase-1 gene via the stress response element pathway. Curr Pharm Des. 2003;9(30):2499-2511.

54. Mace TA, et al. Pancreatic cancer-associated stellate cells promote differentiation of myeloid-derived suppressor cells in a STAT3-dependent manner. Cancer Res. 2013;73(10):3007-3018.

55. Wang Q, et al. Vascular niche IL-6 induces alternative macrophage activation in glioblastoma through HIF-2 $\alpha$. Nat Commun 2018;9(1):559.

56. Bolpetti A, Silva JS, Villa LL, Lepique AP. Interleukin-10 production by tumor infiltrating macrophages plays a role in Human Papillomavirus 16 tumor growth. BMC Immunol. 2010;11:27.

57. Daurkin I, et al. Tumor-associated macrophages mediate immunosuppression in the renal cancer microenvironment by activating the 15-lipoxygenase-2 pathway. Cancer Res. 2011;71(20):6400-6409.

58. Corzo CA, et al. HIF-1 $\alpha$ regulates function and differentiation of myeloid-derived suppressor cells in the tumor microenvironment. J Exp Med. 2010;207(11):2439-2453.

59. Colegio OR, et al. Functional polarization of tumour-associated macrophages by tumour-derived lactic acid. Nature. 2014;513(7519):559-563.

60. Ryter SW, Alam J, Choi AM. Heme oxygenase-1/carbon monoxide: from basic science to therapeutic applications. Physiol Rev. 2006;86(2):583-650.

61. Yin T, et al. Extravascular red blood cells and hemoglobin promote tumor growth and therapeutic resistance as endogenous danger signals. J Immunol. 2015;194(1):429-437.

62. Muliaditan T, et al. Repurposing tin mesoporphyrin as an immune checkpoint inhibitor shows therapeutic efficacy in preclinical models of cancer. Clin Cancer Res. 2018;24(7):1617-1628.

63. Zhang M, et al. Myeloid HO-1 modulates macrophage polarization and protects against ischemia-reperfusion injury. JCI Insight. 2018;3(19):120596.

64. Choi KM, et al. CD206-positive M2 macrophages that express heme oxygenase-1 protect against diabetic gastroparesis in mice. Gastroenterology. 2010;138(7):2399-2409.

65. Gobert AP, et al. Heme oxygenase-1 dysregulates macrophage polarization and the immune response to Helicobacter pylori. J Immunol. 2014;193(6):3013-3022.

66. Tzima S, Victoratos P, Kranidioti K, Alexiou M, Kollias G. Myeloid heme oxygenase-1 regulates innate immunity and autoimmunity by modulating IFN-beta production. J Exp Med. 2009;206(5):1167-1179.

67. Becker T, Zu Vilsendorf AM, Terbish T, Klempnauer J, Jörns A. Induction of heme oxygenase-1 improves the survival of pancreas grafts by prevention of pancreatitis after transplantation. Transplantation. 2007;84(12):1644-1655.

68. Zhao M, Yang M, Que W, Zhong L, Fujino M, Li XK. Myeloid heme oxygenase-1: a new therapeutic target in anti-inflammation. Front Biosci (Landmark Ed). 2018;23:2001-2015.

69. Etzerodt A, Moestrup SK. CD163 and inflammation: biological, diagnostic, and therapeutic aspects. Antioxid Redox Signal. 2013;18(17):2352-2363.

70. Marigo I, et al. T cell cancer therapy requires CD40-CD40L activation of tumor necrosis factor and inducible nitric-oxidesynthase-producing dendritic cells. Cancer Cell. 2016;30(3):377-390.

71. Langmead B, Salzberg SL. Fast gapped-read alignment with Bowtie 2. Nat Methods. 2012;9(4):357-359.

72. Langmead B, Trapnell C, Pop M, Salzberg SL. Ultrafast and memory-efficient alignment of short DNA sequences to the human genome. Genome Biol. 2009;10(3):R25.

73. Zhang Y, et al. Model-based analysis of ChIP-Seq (MACS). Genome Biol. 2008;9(9):R137.

74. Quinlan AR, Hall IM. BEDTools: a flexible suite of utilities for comparing genomic features. Bioinformatics. 2010;26(6):841-842.

75. Love MI, Huber W, Anders S. Moderated estimation of fold change and dispersion for RNA-seq data with DESeq2. Genome Biol. 2014;15(12):550

76. Ramírez F, Dündar F, Diehl S, Grüning BA, Manke T. deepTools: a flexible platform for exploring deep-sequencing data. Nucleic Acids Res. 2014;42(Web Server issue):W187-W191.

77. Butler A, Hoffman P, Smibert P, Papalexi E, Satija R. Integrating single-cell transcriptomic data across different conditions, technologies, and species. Nat Biotechnol. 2018;36(5):411-420.

78. Aibar S, et al. SCENIC: single-cell regulatory network inference and clustering. Nat Methods. 2017;14(11):1083-1086. 